\title{
Melanocortin 1 Receptor Activation Protects Against Alpha-Synuclein Pathologies in Models of Parkinson's Disease
}

\section{Waijiao Cai}

Massachusetts General Hospital https://orcid.org/0000-0003-4730-1145

\section{Danielle Feng}

Massachusetts General Hospital

Yue Lin

Massachusetts General Hospital

Charles R Vanderburg

Massachusetts General Hospital

\section{Yuehang Xu}

Massachusetts General Hospital

\section{Pamela Mclean}

Mayo Clinic: Mayo Clinic's Campus in Florida

Matthew P Frosch

Massachusetts General Hospital

\section{David E Fisher}

Massachusetts General Hospital

Michael A Schwarzschild

Massachusetts General Hospital

Xiqun Chen ( $\nabla$ xchen17@mgh.harvard.edu )

Massachusetts General Hospital https://orcid.org/0000-0001-8582-8891

\section{Research article}

Keywords: Melanocortin 1 receptor, alpha-synuclein, Parkinson's disease, melanoma, nuclear factor erythroid 2-related factor 2

Posted Date: March 13th, 2021

DOl: https://doi.org/10.21203/rs.3.rs-286792/v1

License: (c) (1) This work is licensed under a Creative Commons Attribution 4.0 International License. 
Version of Record: A version of this preprint was published at Molecular Neurodegeneration on February 23rd, 2022. See the published version at https://doi.org/10.1186/s13024-022-00520-4. 


\section{Melanocortin 1 receptor activation protects against alpha-synuclein pathologies in models of Parkinson's disease}

Waijiao Cai ${ }^{1}$, Danielle Feng ${ }^{1}$, Yue Lin ${ }^{1}$, Charles R. Vanderburg ${ }^{1,2}$, Yuehang $\mathrm{Xu}^{1}$, Pamela Mclean ${ }^{3}$, Matthew P. Frosch ${ }^{1,2,4}$, David E. Fisher ${ }^{5}$, Michael A. Schwarzschild ${ }^{1}$, Xiqun Chen $^{1 *}$

\footnotetext{
${ }^{1}$ MassGeneral Institute for Neurodegenerative Disease, Department of Neurology, Massachusetts General Hospital, Harvard Medical School, Boston, USA.

${ }^{2}$ Harvard NeuroDiscovery Advanced Tissue Resource Center, Massachusetts General Hospital, Harvard Medical School, Boston, USA.

${ }^{3}$ Mayo Clinic, Jacksonville, Florida, USA.

${ }^{4}$ Neuropathology Service, Massachusetts General Hospital, Harvard Medical School, Boston, USA.

${ }^{5}$ Cutaneous Biology Research Center, Department of Dermatology, Massachusetts General Hospital, Harvard Medical School, Boston, USA.

${ }^{*}$ Corresponding author: Xiqun Chen

Email: xchen17@mgh.harvard.edu

Author Name Email

Waijiao Cai caiwaijiao@gmail.com

Danielle Feng Danielle.feng@gmail.com

Yue Lin yuelin@bu.edu

Charles R. Vanderburg cvanderb@broadinstitute.org

Yuehang Xu XUY@helix.mgh.harvard.edu

Pamela Mclean McLean.Pamela@mayo.edu

Matthew P. Frosch mfrosch@partners.org

David E. Fisher dfisher3@partners.org

Michael A. MICHAELS@helix.mgh.harvard.edu

Schwarzschild

Xiqun Chen $\quad \underline{x c h e n 17 @ m g h . h a r v a r d . e d u ~}$
} 


\begin{abstract}
Background: Epidemiological studies suggest a link between the melanoma-related pigmentation gene melanocortin 1 receptor $(M C 1 R)$ and risk of Parkinson disease (PD). We previously showed that MC1R signaling can facilitate nigrostriatal dopaminergic neuron survival. The present study investigates the neuroprotective potential of MC1R against neurotoxicity induced by alphasynuclein (aSyn), a key player in PD genetics and pathogenesis.
\end{abstract}

Methods: Nigral dopaminergic neuron toxicity induced by local overexpression of aSyn was assessed in mice that have an inactivating mutation of $M C 1 R$, overexpress its wild-type transgene, or were treated with MC1R agonists. The role of nuclear factor erythroid 2-related factor 2 (Nrf2) in MC1R-mediated protection against aSyn was characterized in vitro. Furthermore, MC1R expression was determined in human postmortem midbrains from patients with PD and unaffected subjects.

Results: Targeted expression of aSyn in the nigrostriatal pathway induced exacerbated synuclein pathologies in $M C 1 R$ mutant mice, which were accompanied by neuroinflammation and altered Nrf2 responses, and reversed by the human MC1R transgene. Two MC1R agonists were neuroprotective against aSyn-induced dopaminergic neurotoxicity. In vitro experiments showed that Nrf2 was a necessary mediator of MC1R effects. Lastly, MC1R was present in dopaminergic neurons in the human substantia nigra and appeared to be reduced in PD patients.

Conclusion: Our study supports an interaction between MC1R and $\alpha$ Syn that can be mediated by neuronal MC1R through Nrf2. It provides evidence for MC1R as a therapeutic target and a rationale for development of MC1R-activating strategies for PD.

\title{
Keywords
}

Melanocortin 1 receptor, alpha-synuclein, Parkinson's disease, melanoma, nuclear factor erythroid 2-related factor 2 


\section{Background}

Parkinson's disease (PD) is a common neurodegenerative disorder and a leading cause of longterm disability. Although symptomatic treatments are available and effective, at least partially, there is currently no therapy known to reverse, arrest, or slow its progressive course. Multiple genetic and environmental factors contribute to the development of PD; among them, alpha-synuclein (aSyn, encoded by SNCA) plays a central role in PD genetics and pathogenesis[1,2]. Mutation in SNCA can cause PD, and accumulation and aggregation of aSyn within Lewy bodies and Lewy neurites in the nervous system are a pathological hallmark of PD. Various cellular events including proteinopathy, neuroinflammation, and oxidative stress contribute to the degenerative process, leading to the eventual loss of dopaminergic neurons of the nigrostriatal dopaminergic pathway of the brain, another pathological hallmark of $\mathrm{PD}[3]$. As proteostasis, the redox system, and inflammatory processes in PD can be orchestrated by nuclear factor erythroid 2-related factor 2 (Nrf2), activation of Nrf2 is a promising therapeutic approach for neurodegenerative disease $[4,5]$.

Melanocortin 1 receptor $(M C 1 R)$ is the major genetic determinant of hair color. Binding of its ligand alpha-melanocyte stimulating hormone $(\alpha-M S H)$ to $M C 1 R$ on melanocytes facilitates brown/black eumelanin synthesis and increases the ratio of yellow/red pheomelanin to eumelanin $[6,7]$. Severe loss-of-function polymorphisms of $M C 1 R$ contributes to red hair/fair skin and are associated with skin aging, and melanoma risk[8-10]. More recent studies identify a critical role of MC1R in regulating physiological functions in the skin including the immune response, DNA repair, and cell differentiation and proliferation, which can be pigmentation-dependent or -independent[8,11]. In addition to its cutaneous expression and function, MC1R is expressed in other tissue and cell types, including immune and endothelial cells, and can modulate the immune system and inflammatory response[12,13]. $\alpha-\mathrm{MSH}$ or its synthetic analog $\mathrm{Nle}^{4}, \mathrm{D}-\mathrm{Phe} \mathrm{P}^{7}-\alpha-\mathrm{MSH}$ (NDP-MSH), a tanning agent and drug approved by the European Medicines Agency for treating the photosensitive skin condition erythropoietic porphyria[14], exerts protective effects in models of ischemic stroke, traumatic brain injury, spinal cord injury, Alzheimer's disease, and neuroinflammatory disease[1518], with MC1R engagement shown to mediate this protection in the latter model[18]. 
Prompted by well-documented epidemiological associations between MC1R, pigmentation, and melanoma, between melanoma and PD as well as possible associations between red hair and PD, and between MC1R variants and PD[19], we previously demonstrated the presence of MC1R in dopaminergic neurons in the mouse substantia nigra (SN) and its influence on dopaminergic neuron survival[20]. Here, we report MC1R-specific protection against aSyn oligomerization and related inflammation and dopaminergic neurotoxicity. In vitro analyses revealed that MC1R counteracted aSyn oligomerization by activating Nrf2. Further, we demonstrate that PD patients exhibited reduced levels of MC1R in the SN.

\section{Methods}

\section{Study design}

The objective of this study was to characterize the protective role of MC1R in the nigrostriatal dopaminergic pathway and to elucidate responsible downstream mediators. Complementary genetic and pharmacological approaches were employed to manipulate MC1R in vivo and in vitro. aSyn pathologies, neuroinflammation, and related dopaminergic neurotoxicity were induced by overexpressing human wild-type (WT) aSyn in mice, HEK cells and primary neuronal cultures. Human studies entailed assessment of postmortem brain tissue from PD patients and controls.

Sample sizes for animal experiments were determined based on our previous studies[20,21] in which significant differences in primary outcome measures (nigral dopaminergic cell counts and striatal dopamine content) were observed. For all animal experiments involving genetic modification of $M C 1 R$ and quantification of the outcome measures, littermates were used as controls. For animal experiments using commercially obtained mice, grouping was randomized. Cell experiments were repeated at least three times with at least three replicates within each condition. Investigators were blind to treatment assignments and/or sample group information wherever practical. All animal and human study protocols were approved by the responsible authorities at Massachusetts General Hospital.

\section{Experimental animals}


$M C 1 R$ extension $\left(M C 1 R^{\mathrm{e} / \mathrm{e}}\right)$ mice carrying an inactivating frameshift mutation of $M C 1 R$ in a C57BL/6J background[20,22] were backcrossed with C57BL/6J mice from the Jackson Laboratory (Bar Harbor, ME). Offspring heterozygous breeders were crossed with each other to generate $M C 1 R^{\mathrm{e} / \mathrm{e}}$ and littermate WT mice.

$M C 1 R$ transgenic $(\mathrm{Tg})$ mice in an e/e background $\left(M C 1 R^{e / e} \mathrm{Tg}\right)$ were originally generated and characterized at University of Edinburgh, UK[23]. $M C 1 R^{\mathrm{e} / \mathrm{e}} \mathrm{Tg}$ mice express the human $M C 1 R$ under the transcriptional control of its human promoter, yielding a physiological expression pattern similar to that in humans. The transgene rescues the $M C 1 R$ deficiency dermal phenotype to give $M C 1 R^{\mathrm{e} / \mathrm{T}} \mathrm{Tg}$ mice a WT-like dark coat. $M C 1 R^{\mathrm{e} / \mathrm{T}} \mathrm{Tg}$ mice were crossed with $M C 1 R^{\mathrm{e} / \mathrm{e}}$ mice to generate $M C 1 R^{\mathrm{e} / \mathrm{e}} \mathrm{Tg}$ and littermate $M C 1 R^{\mathrm{e} / \mathrm{e}}$ mice.

To test the effects of the MC1R agonist BMS-470539, 3-month-old male C57BI/6J mice were purchased from the Jackson Laboratory. To test the effects of the MC1R agonist NDP-MSH, $M C 1 R^{e / e}$ mice and their WT littermates were used.

Mice were maintained in home cages at a constant temperature with a 12-h light/dark cycle and free access to food and water.

\section{Viral vectors and intra-SN infusion}

Vector production and stereotaxic virus intra-SN infusion were described previously[24]. The vectors used were: (1) p adeno-associated virus (AAV)-CBA-human aSyn-WPRE (aSyn AAV), (2) pAAV-CBA-WPRE empty vector (vector), (3) pAAV-CBA-venus1-human aSyn-WPRE and pAAVCBA-human aSyn-venus2-WPRE bimolecular fluorescence complementation (BiFC aSyn AAV), and (4) pAAV-CBA-Venus-WPRE (venus).

Viral vectors were infused at a volume of $2 \mu$ into the left $S N$ at the following coordinates: AP +0.9 $\mathrm{mm}, \mathrm{ML}+1.2 \mathrm{~mm}$, and $\mathrm{DV}-4.3 \mathrm{~mm}$ relative to lambda.

\section{MC1R agonist treatments}


NDP-MSH and BMS-470539 dihydrochloride were purchased from Tocris Bioscience (Bristol, UK). BMS-470539 $(20 \mathrm{mg} / \mathrm{kg})$ or vehicle saline was administered subcutaneously daily starting 1 day after aSyn AAV or empty vector infusion for 4 weeks. A total dose of 3 nmol NDP-MSH in $2 \mu$ PBS was injected intracranially at $30 \mu \mathrm{l} / 60$ min into the left striatum (coordinates: $A P+0.9 \mathrm{~mm}, \mathrm{ML}+2.2$ $\mathrm{mm}$, and DV $-2.5 \mathrm{~mm}$ relative to bregma). Control mice received PBS injection. aSyn AAV was infused into the SN immediately after NDP-MSH or vehicle administration.

\section{Sequential tissue extraction and aSyn immunoblotting}

Mice were sacrificed, and their ventral midbrain and striatum were dissected. Protein sequential extraction and immunoblotting of aSyn were conducted as previously reported[24] with modifications. Briefly, tissues were homogenized in 1\% Triton X-100 buffer and centrifuged. The supernatant was designated as the "Triton X-100-soluble" fraction. The pellet was resuspended in lysis buffer containing $2 \%$ SDS and designated as the "SDS-soluble" fraction. Protein concentrations were determined by BCA protein assay. Protein from each Triton X-100-soluble (50 $\mu \mathrm{g})$ and SDS-soluble $(80 \mu \mathrm{g})$ sample were run on Nupage 4-12\% SDS-PAGE gel and transferred to PVDF membranes following fixation with $0.4 \%$ paraformaldehyde for $30 \mathrm{~min}$. Primary antibody against human aSyn (clone Syn211, ThermoFisher Scientific, AHB0261) was added at 1:700 and incubated overnight at $4^{\circ} \mathrm{C}$. Membranes were then incubated with a secondary antibody. Signals were detected using enhanced chemiluminescence. Band densities were determined using ImageJ and normalized to ponceau staining.

\section{Immunostaining, imaging, and quantification}

Mice were sacrificed, and their brains were processed and sectioned coronally as described[24]. For immunostaining, sections were incubated with primary antibodies overnight at $4^{\circ} \mathrm{C}$ and corresponding secondary antibodies for $1 \mathrm{~h}$ at $37^{\circ} \mathrm{C}$. The primary antibodies used were against human aSyn (clone Syn211, ThermoFisher Scientific, AHB0261) at 1:500, phosphorylated aSyn at serine 129 (p-aSyn) (p-syn/81A, BioLegend, 825701) at 1:500, glial fibrillary acidic protein (GFAP) (clone GA5, Sigma, G3893 and MAB360) at 1:1000, ionized calcium binding adapter molecule 1 
(iba1) (clone EPR16588, Abcam, ab178846, and ab107159) at 1:500, tyrosine hydroxylase (TH) (clone TH2, Sigma, T1299) at 1:1000, and Nrf2 (Abcam, ab31163) at 1:500. For fluorescence staining, sections were incubated with goat anti-rabbit or anti-mouse IgG-Alexafluor-546 or -488 . For DAB staining, sections were incubated with appropriate secondary antibodies, and staining was developed by incubating with DAB.

MC1R staining was performed as previously reported[20] with modifications. Sections were heated for antigen retrieval[25] and incubated with primary anti-MC1R (Santa Cruz, SC-19485) at 1:50 or anti-human MC1R (LSBio LS-A1040) at 1:100 overnight at $4^{\circ} \mathrm{C}$. Sections were then incubated with Alexa Fluor conjugated secondary antibody at $1: 200$ at $37 \stackrel{\circ}{ } \mathrm{C}$ for $30 \mathrm{~min}$. After washes, subsequent TH, GFAP, or iba1 staining was performed.

Fluorescence images were captured under a Nikon C2s laser scanning microscope. Images from DAB-stained sections were captured under an Olympus BX50 microscope with a DP 70 digital camera system. Posterior (interaural 0.00/bregma $-3.80 \mathrm{~mm}$ ), posterior central (interaural 0.28 $\mathrm{mm} /$ bregma $-3.52 \mathrm{~mm}$ ), anterior central (interaural $0.64 \mathrm{~mm} /$ bregma $-3.16 \mathrm{~mm}$ ), and anterior (interaural $0.88 \mathrm{~mm} /$ bregma $-2.92 \mathrm{~mm}$ ) midbrain sections from each mouse[26] were selected for quantification unless stated otherwise.

To evaluate aSyn transduction efficiency, midbrain sections were co-labeled with antibodies against human $\alpha$ Syn and TH. To determine the percentage of aSyn-positive dopaminergic neurons in the SN, images were acquired in 488- and 546-nm channels with $40 \times$ magnification. ImageJ software was used to count TH-positive cells and cells that were both $\mathrm{TH}$ - and aSyn-positive.

Quantification of $p$-aSyn staining was performed using the optical fractionator method at $40 x$ magnification (Olympus BX51 microscope and Olympus CAST stereology software)[27] to count positively stained particles in the SN.

For astrogliosis and microgliosis analyses, GFAP staining and the morphology of iba1-positive cells in the SN pars compacta (SNpc) were analyzed as previously described[24]. 
For proteinase $\mathrm{K}$ digestion, sections were mounted onto slides, dried overnight, and coverslipped[24]. After image acquisition, cover slips were carefully removed, and sections were rehydrated. Sections were incubated with $50 \mu \mathrm{g} / \mathrm{ml}$ proteinase $\mathrm{K}$ at $55^{\circ} \mathrm{C}$ for $120 \mathrm{~min}$, and images were recaptured. Reconstituted venusYFP intensity was quantify using ImageJ.

For thioflavin-S staining and quantification, sections were incubated with $0.05 \%$ thioflavin S solution for $8 \mathrm{~min}[28]$. Images were recaptured in $488-\mathrm{nm}$ channel at $40 \times$ magnifications. Thioflavin-S fluorescence intensity was quantified using ImageJ.

To assess nuclear-to-cytoplasmic Nrf2 ratio, sections were counterstained by DAPI to reveal the nucleus, and nuclear Nrf2 signal was defined within DAPI regions. Sections were imaged at excitations of $488 \mathrm{~nm}$ for Nrf2 and $568 \mathrm{~nm}$ for $\mathrm{TH}$. The mean fluorescence intensities of nuclear and cytoplasmic Nrf2 per cell were measured, and the ratio was determined using ImageJ's "Intensity Ratio Nuclei Cytoplasm Tool" as previously described[29]. Only TH-positive neurons were measured. Five to ten cells were randomly picked on each side of the SN from each section and a total of 30 cells on each side from each animal were analyzed.

\section{Protein oxidation}

Protein carbonyls in ventral midbrain tissue were detected using an Oxyblot protein oxidation detection kit (Millipore, S7150) according to the manufacturer's instructions. Band density was analyzed using ImageJ and normalized to ponceau staining density.

\section{Nrf2 Western blotting}

Ventral midbrain tissues were lysed, and proteins were extracted and electrophoresed. The blot was probed with anti-Nrf2 (Abcam, ab31163) at 1:1000. Band density was analyzed using ImageJ and normalized by actin.

\section{Quantitative polymerase chain reaction (qPCR) for cytokines and Nrf2 target genes}

Total RNA was isolated using TRI reagent (Invitrogen) and reverse-transcribed into cDNA using a superscript III kit (Invitrogen). qPCR was performed in a 96-well plate using SYBR Green PCR 
Master Mix in an Applied Biosystem 7500. GAPDH was used to normalize expression levels of the target genes. The $2^{-\Delta \Delta C t}$ method was employed for data analysis[30]. The primers used are provided in Supplementary Table 1.

\section{Amphetamine-induced rotational behavior}

Amphetamine-induced (5 mg/kg, intraperitoneal) rotational behavior was assessed by an automated rotometry system (San Diego Instruments) for $60 \mathrm{~min}$ as previously described[24,31].

\section{Striatal dopamine measurement}

Mice were sacrificed, and the striatum was dissected. Dopamine content was determined by highperformance liquid chromatography (HPLC) coupled with electrochemical detection as previously described[24,31].

\section{Stereological analysis of SN dopaminergic neurons}

A complete set of serial midbrain sections were collected. immunostained for $\mathrm{TH}$ and counterstained for Nissl to reveal dopaminergic neurons and total neurons. Unbiased stereological counting was performed as previously described[24,31].

HEK293T cell transfection and transduction, immunostaining and quantification, and cell assays

HEK293T cells were purchased from Clonetech. Cells were maintained in DMEM with 10\% FBS in a humidified incubator at $37^{\circ} \mathrm{C}$ with $5 \% \mathrm{CO}_{2}$. pcDNA3.1(+)-human WT aSyn and control plasmid were provided by former colleague Dr. Joseph Mazzulli (Northwestern University, IL)[32]. MC1RTango expressing human MC1R tagged with FLAG and vector control GPRC5A-Tango were gifts from Bryan Roth (Addgene plasmid \#66427 and \#66382). aSyn and MC1R and their respective controls were transfected into cells using Lipofectamine ${ }^{\circledR} 2000$ (ThermoFisher Scientific) according to the manufacturer's instructions. 
Human shNrf2 was purchased from Dharmacon RNAi Consortium (RHS4533-EG4780). pLKO.1scrambled RNA (scRNA) (Sigma, SHC016-1EA) was used as a control. Plasmids were packaged in lentivirus with packaging plasmid psPAX2 and envelope plasmid pMD2.G (Addgene plasmid \#12259 and \#12260, gifts from Didier Trono). Lentiviral particles were produced in HEK293T cells. For scRNA or shNrf2 transduction, cells were incubated in medium containing lentiviral particles in the presence of polybrene for $16 \mathrm{~h}$.

Cells were harvested $48 \mathrm{~h}$ after transfection with or without viral transduction and lysed for immunoblotting or qPCR. For aSyn immunoblotting, in-cell crosslinking was performed using disuccinimidyl suberate ligand (ThermoFisher Scientific) as previously described[33]. Primary antibodies used were anti-human aSyn (clone Syn211, ThermoFisher Scientific, AHB0261) at 1:500, anti-Nrf2 (Abcam, ab31163) at 1:1000, anti-FLAG (Sigma, F1804) at 1:1000, and antiMC1R (LSBio LS-A1040) at 1:1000. Densities of bands were analyzed using ImageJ and normalized by actin. The primers used are provided in Supplementary Table 1.

For immunocytochemistry, cells were grown on pre-coated poly-lysine coverslips, fixed $48 \mathrm{~h}$ posttransfection with or without viral transduction in 4\% PFA for $10 \mathrm{~min}$, and blocked in $5 \%$ normal goat serum in PBS/0.3\% Triton X-100 for 30 min at $37 \stackrel{\circ}{ }$ C. Cells were incubated with anti-Nrf2 (Abcam, ab31163, 1:200) overnight at $4^{\circ} \mathrm{C}$ and Alexa-Fluor-488 for $1 \mathrm{~h}$ at $37^{\circ} \mathrm{C}$. Images were acquired under a Nikon C2s laser scanning microscope. The subcellular distribution of Nrf2 fluorescence in nuclear and cytoplasmic regions was quantified using ImageJ as described above and previously in cell cultures[29].

\section{Primary neuron cultures, viral transduction, NDP-MSH treatment, immunostaining and quantification, and cell assays}

Primary cortical neurons were prepared as previously described $[34,35]$ from the cerebral cortex of embryonic day 16-17 WT and $M C 1 R^{\mathrm{e} / \mathrm{e}}$ mice. Mouse shNrf2 was purchased from Sigma (SHCLNG, NM 010902) and packaged with lentivirus. For lentiviral transduction, cells were incubated in 250 $\mu \mathrm{l}$ medium containing lentiviral scRNA or shNrf2 and $250 \mu \mathrm{l}$ complete neuron growth medium 
(Neurobasal medium with 2\% B27 supplement, $2 \mathrm{mM} \mathrm{L-gluatamine,} 100 \mathrm{U} / \mathrm{ml}$ penicillin, and 100 $\mu \mathrm{g} / \mathrm{ml}$ streptomycin) in the presence of polybrene at DIV3 for $48 \mathrm{~h}$ in a 24 -well plate. BiFC aSyn AVV or control venus AAV was added at $1.25 \times 10^{10} \mathrm{gc}$ to each well for $48 \mathrm{~h}$. Medium was changed to complete neuron growth medium supplemented with or without $10 \mathrm{nM} \mathrm{NDP-MSH}$ at DIV5. At DIV12, $50 \mu$ l supernatant from each well was taken for lactate dehydrogenase (LDH) release assay using a Pierce LDH Cytotoxicity Assay Kit (ThermoFisher Scientific 88953, 88954) according to the manufacturer's instructions. Nrf2 knock-down efficiency was determined by qPCR.

At DIV9, images of living neurons were obtained under an inverted Leica fluorescence microscope. At DIV12, neurons transduced with aSyn AAV or control venus AAV were fixed and immunostained. Primary antibodies used were against MAP2 (Invitrogen, PA5-17646, or 13-1500 for double staining) at 1:250, MC1R (Santa Cruz, SC-19485) at 1:1000, and human aSyn (Thermo Fisher Scientific, AHB0261) at 1:250. Cell were counterstained by DAPI.

For MAP2-positive cell counting, three images were captured randomly from each coverslip under a 20x objective in an Olympus BX50 microscope equipped with a DP70 digital camera system. MAP2 and DAPI channels were merged, and MAP2-positive cells in each visual field were counted using ImageJ[36]. A total of nine images from three replicate coverslips were analyzed.

\section{Human samples}

Postmortem frozen SN tissue from 5 pathological diagnosed PD patients (male=3, female=2) and 4 control individuals with no neurological conditions (male $=3$, female $=1$ ) was obtained from the Massachusetts Alzheimer's Disease Research Center of the Massachusetts General Hospital. Average age at death was $75.3 \pm 8.0$ years for controls and $80.8 \pm 6.7$ years for PD patients. Average postmortem interval $(\mathrm{PMI})$ was $21.8 \pm 8.9 \mathrm{~h}$ for controls and $19.4 \pm 5.6 \mathrm{~h}$ for PD patients. All subjects were Caucasian. Tissues were cryosectioned at $8 \mu \mathrm{m}$ and airdried at $37^{\circ} \mathrm{C}$ for $2 \mathrm{~min}$ and fixed in $70 \%$ ethanol for 2 min. For peroxidase immunostaining, sections were immersed in $1 \% \mathrm{H}_{2} \mathrm{O}_{2}$ in $100 \%$ methanol for $10 \mathrm{~min}$ at $37 \stackrel{\circ}{\circ}$ to quench endogenous peroxidase. Sections were then blocked by $5 \%$ normal goat serum and incubated with anti-human MC1R (LSBio, LS-A1040) at 1:50 and/or 
anti-TH (Sigma, T1299) at 1:500 at $37^{\circ} \mathrm{C}$ for $45 \mathrm{~min}$. After three washes, sections were incubated with either biotin-conjugated secondary antibody at 1:500 for peroxidase immunostaining or Alexa Fluor-conjugated secondary antibodies at 1:200 for fluorescent immunostaining at $37^{\circ} \mathrm{C}$ for 30 $\min$.

For immunoblot, twenty sections $(10 \mu \mathrm{m})$ containing only $\mathrm{SN}$ from frozen tissues were collected in RIPA lysis buffer. Protein sample $(120 \mu \mathrm{g})$ was loaded and run on Nupage $4-12 \%$ SDS-PAGE gels at 150 voltage for $80 \mathrm{~min}$ and transferred to PVDF membranes at 90 voltage for $75 \mathrm{~min}$ at $4{ }^{\circ} \mathrm{C}$. Blots were then probed by anti-human MC1R (LSBio LS-A1040) at 1:700 and anti TH (Sigma, T1299) at 1:1000 overnight at $4{ }^{\circ} \mathrm{C}$, followed by an extra $1 \mathrm{hr}$ incubation at $37^{\circ} \mathrm{C}$ for MC1R probing to enhance the binding. Actin was used as the loading control. Blots were then incubated with antirabbit or anti-mouse secondary antibodies at 1: 1000 for $1 \mathrm{hr}$ at $37^{\circ} \mathrm{C}$. Blots were incubated in enhanced chemiluminescence for 2 min and then scanned by a LI-COR Odyssey Fc Image System for $10 \mathrm{~min}$.

All human tissue study protocols were approved by the Partners Human Research Committee.

\section{Statistical analysis}

All values are presented as the mean \pm SEM. Pairwise comparisons were performed using unpaired two-tail Student's $t$ test. Multiple comparisons were performed using ANOVA and Tukey's post hoc test. $P$-values $\leq 0.05$ were considered statistically significant.

\section{Results}

MC1R disruption exacerbates synucleinopathies in the nigrostriatal pathway in aSyn AAVinjected mice

Our previous study demonstrates that the nigrostriatal dopaminergic pathway is compromised under basal conditions in $M C 1 R^{\mathrm{e} / \mathrm{e}}$ mice carrying a loss-of-function gene mutation and exhibiting blond-red fur[20]. To further investigate how MC1R defends the dopaminergic pathway against aSyn, we injected human WT aSyn AAV into the SN of 5-6-month-old $M C 1 R^{\mathrm{e} / \mathrm{e}}$ mice and their WT littermates[24]. Expression of human aSyn was detected in the ipsilateral SN 4 weeks post-injection 
(Fig. S1A). Quantification of fluorescence double-labeled for human aSyn and TH, a marker of dopaminergic neurons, showed $70 \%$ and $67 \%$ transduction efficiencies for SN dopaminergic neurons in WT and $M C 1 R^{e / e}$ mice, respectively (Fig. S1A, B). By contrast, only few astrocytes in the $\mathrm{SN}$ were transduced on the ipsilateral side in $\mathrm{WT}$ and $M C 1 R^{\mathrm{e} / \mathrm{e}}$ mice as shown by doublelabeling for human aSyn and GFAP, a marker of glial cells (Fig. S1C). Similarly, there was almost no colocation of aSyn and iba1, a marker of microglia, 4 weeks post-injection (Fig. S1D). These results are consistent with the reported neural tropism of AAV8[37,38].

High molecular weight aSyn species were detected by sequential tissue extraction followed by Western blotting using an antibody against human aSyn 8 weeks after AAV injection. Consistent with our previous results, aSyn overexpression in WT mice was associated with aSyn aggregation in the ventral midbrain and striatum on the injection side[24]. Higher molecular weight aSyn species were significantly more abundant in both Triton-soluble and -insoluble SDS-soluble fractions from the injection side in $M C 1 R^{e / e}$ mice compared with WT mice (Fig. 1A, B). Profound p-aSyn, which is associated with aging and synucleinopathies[39,40], was detected by immunohistochemistry in the ipsilateral SN in $M C 1 R^{e / e}$ mice. Quantification showed increased p-aSyn accumulation in the SN of $M C 1 R^{\mathrm{e} / \mathrm{e}}$ mice compared with WT mice (Fig. 1C, D).

A seperate set of $M C 1 R^{e / e}$ and WT mice received injections of human WT aSyn AAV fused to the $\mathrm{N}$-terminus and $\mathrm{C}$-terminus halves of venus YFP into the $\mathrm{SN}$, and aSyn aggregation was visualized by conjugated fluorescence. Our previous study demonstrated that the dopaminergic phenotype induced by this BiFC aSyn is comparable to that in non-BiFC aSyn mouse models[24]. Profound reconstituted YFP fluorescence was observed 8 weeks after AAV injection (Fig. 1E, F). Fluorescence intensity was higher in the ipsilateral $S N$ of $M C 1 R^{e / e}$ mice than in that of WT mice (Fig. 1E, F). Proteinase $\mathrm{K}$ digestion revealed the soluble nature of the vast majority of aSyn aggregates. However, $M C 1 R^{\mathrm{e} / \mathrm{e}}$ mice had significantly higher levels of proteinase K-resistant aSyn aggregates than WT mice (Fig. 1E, F). Thioflavin-S dyeing provided further evidence of more amyloid fibrils in the ipsilateral $S N$ in $M C 1 R^{e / e}$ mice compared with WT mice (Fig. 1G, H). Collectively, these results show exacerbated synucleinopathies in the nigrostriatal pathway 
resulting from $M C 1 R$ loss of function in a aSyn AAV mouse model. No human aSyn transduction or pathology on the contralateral, non-injected side was observed in either $M C 1 R^{\mathrm{e} / \mathrm{e}}$ or WT mice (Fig. S1A).

\section{$M C 1 R$ disruption amplifies aSyn overexpression-induced microglia activation and alters Nrf2 response in the nigrostriatal pathway}

Oxidative stress and activation of the immune system, which involve MC1R signaling $[8,13]$, contribute to neurodegeneration in PD. To determine whether MC1R disruption is associated with altered inflammation and oxidative stress in response to aSyn AAV injection, we assessed microglial cells by immunostaining for iba1 8 weeks after injection. Consistent with our previous report[24], aSyn overexpression increased iba1 immunoreactivity on the ipsilateral side compared with the contralateral side. Also, an overall enhanced iba1 signal was observed in $M C 1 R^{\mathrm{e} / \mathrm{e}}$ mice compared with WT mice (Fig. 2A, B). Iba1-positive cells were classified based on their morphology, and numbers of cell subtypes were counted. Numbers of reactive and phagocytic microglia on the injection side were dramatically increased in $M C 1 R^{\mathrm{e} / \mathrm{e}}$ mice. There were also trends toward increased numbers of resting and reactive microglia on the contralateral, non-injected side in $M C 1 R^{\mathrm{e} / \mathrm{e}}$ mice compared with WT mice, indicating possible basal microgliosis due to $M C 1 R$ disruption. Venus or Vector did not induce microgliosis either in WT or $M C 1 R^{e / e}$ mice (Fig. S2A, C).

Integrated optical density of GFAP staining in the SN revealed increased GFAP immunoreactivity in WT mice (Fig. S3A), consistent with the previously reported astrogliosis following aSyn overexpression by our group and others[24,40]. A similar increase in GFAP immunoreactivity was identified in $M C 1 R^{e / e}$ mice, both in absolute value and after normalization for increased GFAP on the contralateral side (Fig. S3B, C). There were no significant differences on the ipsilateral side between genotypes (Fig. S3C). Venus or Vector did not induce astrogliosis either in WT or MC1 $R^{\mathrm{e} / \mathrm{e}}$ mice (Fig. S2A, B).

mRNA levels of the pro-inflammatory cytokines interleukin (IL)-6, tumor necrosis factor (TNF) $\alpha$, intercellular adhesion molecule 1 (ICAM1), and IL-1 $\alpha$ in the ventral midbrain were analyzed 8 
weeks after AAV injection using qPCR. aSyn overexpression increased levels of IL-6, TNF $\alpha$, and ICAM-1 on the ipsilateral side compared with the contralateral side in both $M C 1 R^{\mathrm{e} / \mathrm{e}}$ and WT mice (not significant for IL-1 $\alpha$ ). Compared with WT mice, $M C 1 R^{\mathrm{e} / \mathrm{e}}$ mice exhibited significantly higher levels of IL-6, TNF $\alpha$, and ICAM1 in the ipsilateral midbrain (Fig. 2C). These results indicate that $M C 1 R$ dysfunction alters the immune response to aSyn.

We previously found increased oxidative protein and DNA damage in the $M C 1 R^{\mathrm{e} / \mathrm{e}}$ mouse brain under basal conditions[20]. Here, we measured protein carbonylation, a general marker of oxidative stress, by Oxyblot in the ipsilateral ventral midbrain of $M C 1 R^{e / e}$ and WT mice injected with aSyn AAV and found more protein carbonyls in $M C 1 R^{e / e}$ mice than in WT mice 8 weeks after injection (Fig. 2D, E).

We next assessed transcription factor Nrf2 signaling, a key regulator of antioxidant and antiinflammatory pathways through its nuclear translocation and activation and subsequent transcriptional activation of target genes[41]. Western blot showed significantly reduced Nrf2 in the ipsilateral ventral midbrain of $M C 1 R^{\mathrm{e} / \mathrm{e}}$ mice 8 weeks after $\alpha \mathrm{Syn}$ AAV injection as compared with WT mice (Fig. 2F, G). Contralateral Nrf2 was also significantly decreased in $M C 1 R^{e / e}$ mice compared with WT mice. Given that no aSyn pathologies were observed on the contralateral side, this difference may reflect compromised Nrf2 signaling due to disrupted MC1R under basal conditions without additional insult. After normalizing for the "basal" difference between genotypes by expressing Nrf2 as a percentage of the contralateral value, aSyn overexpression significantly increased Nrf2 levels on the ipsilateral side in WT mice but not in $M C 1 R^{e / e}$ mice (Fig. 2H).

The localization of Nrf2 in dopaminergic neurons in the $\mathrm{SN}$ was assessed by fluorescence doublelabeling for Nrf2 and TH. Enhanced nuclear localization and accumulation of Nrf2 in dopaminergic

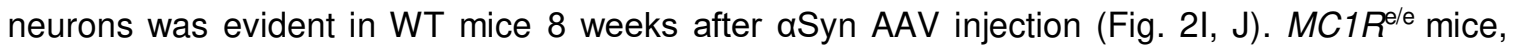
however, displayed a lower ratio of nuclear-to-cytoplasmic Nrf2 in $\mathrm{TH}$-positive neurons following aSyn overexpression compared with WT mice. There was no significant change in the ratio between ipsilateral and contralateral sides in $M C 1 R^{\mathrm{e} / \mathrm{e}}$ mice (Fig. 2J). As expected, the mostly inactive Nrf2 was accompanied by markedly lower mRNA levels of the Nrf2 target genes heme 
oxygenase $1(\mathrm{HO} 1), \mathrm{NAD}(\mathrm{P}) \mathrm{H}$ quinone dehydrogenase (NQO1), and glutamate-cysteine ligase subunit C (GCLC) in the ipsilateral ventral midbrain in $M C 1 R^{\mathrm{e} / \mathrm{e}}$ mice as assessed by $\mathrm{qPCR}$ (Fig. 2K). Taken together, these results demonstrate the absence of Nrf2 activation following aSyn overexpression in terms of both protein induction and nuclear translocation as well as accompanying oxidative damage and an inflammatory response in $M C 1 R^{\mathrm{e} / \mathrm{e}}$ mice.

Astrocytic Nrf2 signaling is considered critical in aging and neurodegeneration[5,42]. However, we did not observe appreciable alteration in Nrf2 immunoreactivity overlapping with GFAP in the SN between the contralateral and ipsilateral sides in either WT or $M C 1 R^{\mathrm{e} / \mathrm{e}}$ mice (Fig. S3D).

\section{MC1R disruption exacerbates aSyn-induced dopaminergic neurotoxicity, which is reversed by human MC1R transgene}

We next assessed dopaminergic neurotoxicity due to aSyn overexpression in $M C 1 R^{\mathrm{e} / \mathrm{e}}$ and WT mice. Six-month-old mice were injected unilaterally with human WT aSyn AAV in the SN. Rotational behavior induced by the dopamine-releasing agent amphetamine was analyzed 12 weeks following AAV injection. $M C 1 R^{\mathrm{e} / \mathrm{e}}$ mice displayed a tendency to rotate toward the ipsilateral side after AAV injection, whereas WT mice tended to rotate toward the contralateral side (Fig. 3A), as previously reported[24]. There was a significant difference in the number of ipsilateral turns between genotypes. When we measured dopamine content in the striatum by HPLC at 16 weeks, we found that aSyn overexpression induced significantly greater DA depletion on the ipsilateral side in $M C 1 R^{e / e}$ mice than in WT mice (Fig. 3B). When we analyzed dopaminergic neuron survival by stereological counting of TH-positive cells in the $\mathrm{SN}$, we found a more substantial and significant loss of dopaminergic neurons in $M C 1 R^{e / e}$ mice compared with WT mice (Fig. 3C, D). Mild-tomoderate reductions in striatal dopamine $(100 \pm 3 v s .111 \pm 2)$ and nigral dopaminergic neuron counts (4543 \pm 628 vs. $6204 \pm 323$ ) on the contralateral side in $M C 1 R^{\mathrm{e} / \mathrm{e}}$ mice are consistent with our previously reported basal dopaminergic defects[20] assuming that the contralateral side is similar to the intact condition, as no contralateral aSyn transduction was detected (Fig. S1A). Representative images of ventral midbrain TH staining show extensive loss of TH-positive cells in a $M C 1 R^{e / e}$ mouse (Fig. 3C). Nissl counterstaining and stereological counting of $\mathrm{TH}$-negative 
neurons in the SNpc revealed no difference between $M C 1 R^{\mathrm{e} / \mathrm{e}}$ and WT mice, suggesting that the deleterious effects of $M C 1 R$ disruption and its interaction with aSyn are dopaminergic-specific, at least in the ventral midbrain (Fig. 3D).

To investigate whether MC1R disruption is causative of higher aSyn susceptibility and to explore the potential of human $M C 1 R$ to reverse the $M C 1 R$ loss-of-function phenotypes, we employed $\mathrm{Tg}$ mice expressing human $M C 1 R$ using the human promoter to simulate physiological expression in humans[23]. The transgene reverses the $M C 1 R$ deficiency yellow/red pigmentation phenotype to give $\mathrm{Tg}$ mice in an e/e background $\left(M C 1 R^{\mathrm{e} / \mathrm{Tg}} \mathrm{T}\right.$ ) a WT-like dark coat. We first confirmed transgene expression in $M C 1 R^{e / e} \mathrm{Tg}$ mice using human MC1R specific antibody. Robust human MC1R was detected in the SN, mostly in the cytoplasm as well as on the cell surface. This transgene expression pattern was similar to that of the endogenous mouse receptor stained using non-human specific antibody in WT adult C57BL/6J mice (Fig. S4A) and is consistent with our previous report[20]. Double-labeling for $\mathrm{TH}$ and $\mathrm{MC} 1 \mathrm{R}$ showed the presence of $\mathrm{Tg} M C 1 \mathrm{R}$ in dopaminergic neurons in the SN (Fig. S4A). Double-labeling for GFAP and MC1R showed that most GFAPpositive cells were in the SN pars reticulata, where only a few cells were MC1R-positive. Few GFAP-positive astrocytes co-expressed Tg human MC1R (Fig. S4B), also similar to the endogenous expression pattern of $\mathrm{MC1R}$ in WT C57BL/6J mice (Fig. S4B). Similarly, there appeared to be only negligible overlap between MC1R and iba1 in the SN in either $M C 1 R^{\mathrm{e} / \mathrm{T}} \mathrm{Tg}$ or WT mice ((Fig. S4C).

We next unilaterally injected 5-6-month-old $M C 1 R^{\mathrm{e} / \mathrm{e}} \mathrm{Tg}$ mice and their $M C 1 R^{\mathrm{e} / \mathrm{e}}$ littermates with aSyn AAV. Similar aSyn transduction efficiency was confirmed on the injection side in $M C 1 R^{e / e} \mathrm{Tg}$ and $M C 1 R^{\mathrm{e} / \mathrm{e}}$ mice by double-staining for human aSyn and TH (Fig. S4D, E) 4 weeks after injection. Double-labeling for human aSyn and GFAP showed that few astrocytes in the SN were transduced in either $M C 1 R^{e / e} \mathrm{Tg}$ or $M C 1 R^{\mathrm{e} / \mathrm{e}}$ mice (Fig. S4F).

Dopaminergic phenotypes were assessed 16 weeks after aSyn AAV injection. $M C 1 R^{\mathrm{e} e} \mathrm{Tg}$ mice showed $33 \%$ dopamine depletion on the ipsilateral side as compared with $59 \%$ dopamine depletion in $M C 1 R^{e / e}$ mice after normalizing to the contralateral sides within each genotype to eliminate the 
likely basal difference between genotypes, similar to the comparison between WT and $M C 1 R^{e / e}$ mice (Fig. 3E). A higher percentage of surviving SN dopaminergic neurons after AAV injection was also observed in $M C 1 R^{\mathrm{e} / \mathrm{e}} \mathrm{Tg}$ mice $(70 \%)$ as compared with $M C 1 R^{\mathrm{e} / \mathrm{e}}$ mice $(51 \%)$ (Fig. 3F, G). Differences in contralateral striatal dopamine level and dopaminergic neuron counts between genotypes likely reflect the restoration of previously reported deficits in $M C 1 R^{\mathrm{e} / \mathrm{e}}$ mice under basal conditions (Fig. 3E-G)[20].

To confirm human $M C 1 R$ rescue of aSyn neurotoxicity in the e/e background, we assessed aSyn pathology, neuroinflammation, and Nrf2 in the SN in $M C 1 R^{\mathrm{e} / \mathrm{T}} \mathrm{Tg}$ and $M C 1 R^{\mathrm{e} / \mathrm{e}}$ mice. Twelve weeks after aSyn AAV injection, $M C 1 R^{\mathrm{e} / \mathrm{T}} \mathrm{Tg}$ mice showed significantly less $\mathrm{p}$ - $\alpha \mathrm{Syn}$ aggregates on the ipsilateral side compared with $M C 1 R^{e / e}$ mice (Fig. $\left.3 \mathrm{H}, \mathrm{I}\right)$. No p-aSyn staining was observed on the contralateral side in either $M C 1 R^{\mathrm{e} / \mathrm{e}} \mathrm{Tg}$ or $M C 1 R^{\mathrm{e} / \mathrm{e}}$ mice. Iba1 immunoreactivity was increased overall on the ipsilateral side compared with the contralateral side in both $M C 1 R^{\mathrm{e} / \mathrm{T}} \mathrm{Tg}$ and $M C 1 R^{\mathrm{e} / \mathrm{e}}$ mice 12 weeks after AAV injection. Quantification of morphology-based cell subtypes revealed a significant reduction in the numbers of reactive and phagocytic microglia in the injected $\mathrm{SN}$ in $M C 1 R^{\mathrm{e} / \mathrm{T}} \mathrm{Tg}$ mice compared with $M C 1 R^{\mathrm{e} / \mathrm{e}}$ mice (Fig. 3J, K).

Double-immunostaining for Nrf2 and TH showed a significantly higher nuclear-to-cytoplasmic Nrf2 ratio on the ipsilateral side in $M C 1 R^{\mathrm{e} / \mathrm{T}} \mathrm{Tg}$ mice compared with $M C 1 R^{\mathrm{e} / \mathrm{e}}$ mice 12 weeks after $\mathrm{AAV}$ injection (Fig. 3L, M). qPCR analysis showed induction of Nrf2 target genes HO-1, NQO1, GCLM, and GCLC at the mRNA level in the ipsilateral ventral midbrain in $M C 1 R^{\mathrm{e} / \mathrm{Tg}} \mathrm{mice}$ compared with $M C 1 R^{e / e}$ mice (Fig. 3N). Nrf2 signal within GFAP-positive cells in the SN did not seem to be prominent on either side in $M C 1 R^{e / e}$ and did not appear to be altered in $M C 1 R^{e / e} \mathrm{Tg}$ mice (Fig. S4G).

\section{Pharmacological MC1R activation is neuroprotective against $\alpha$ Syn-induced dopaminergic neurotoxicity}

This proof-of-concept genetic rescue provides rationale for MC1R as a therapeutic target for PD. We previously reported a dopaminergic neuroprotective effect of systemically administered MC1R agonist BMS-470539 in a MPTP mouse model of PD[20]. BMS-470539 is MC1R-selective and has 
$\sim 10 \%$ brain penetrance. $\mathrm{C} 57 \mathrm{BI} / 6 \mathrm{~J}$ mice were injected with aSyn AAV and treated daily with BMS470539 for 4 weeks at a dose of $20 \mathrm{mg} / \mathrm{kg}$ subcutaneously. aSyn reduced striatal DA on the ipsilateral side in saline-treated mice 16 weeks after AAV injection, whereas BMS-470539 treatment significantly attenuated the aSyn-induced DA deficit (Fig. 4A). Stereological counting of $\mathrm{TH}$-positive cells revealed a significantly higher number of surviving $\mathrm{SN}$ dopaminergic neurons in BMS-470539-treated mice compared with saline-treated mice (Fig. 4B). Reduced p-aSyn aggregates were observed on the ipsilateral side in BMS-470539-treated mice compared with saline-treated mice (Fig. 4C, D). Consistent with our previous report[24] injection of empty vector AAV did not induce significant depletion of striatal dopamine or loss of SN dopaminergic neurons. Moreover, BMS-470539 treatment did not alter striatal dopamine content or SN dopaminergic neuron counts in vector-injected mice (Fig. 4A, B).

We next tested another MC1R agonist, NDP-MSH, in our aSyn AAV mouse model. In a pharmacokinetic study with $\mathrm{C} 57 \mathrm{BI} / 6 \mathrm{~J}$ mice, no NDP-MSH was detected in the brain any time point from 5 to $120 \mathrm{~min}$ after $1 \mathrm{mg} / \mathrm{kg}$ intraperitoneal injection ( $\mathrm{n}=3 /$ time point). NDP-MSH was administered intracranially into the left striatum at $3 \mathrm{nmol}$ before aSyn AAV injection in WT C57BI/6J mice. NDP-MSH treatment mildly but significantly increased striatal dopamine and SN dopaminergic neuron count on the ipsilateral side 16 weeks after AAV injection compared with PBS-treated mice (Fig. 4E, F). When we assessed p-aSyn and iba1 in the ipsilateral SN by immunohistochemistry, we found dramatically reduced p-aSyn in NDP-MSH-treated mice compared with PBS-treated mice (Fig. 4G, H), and this reduction in p-aSyn on the ipsilateral side was accompanied by only modest microglia activation as reflected by a decreased number of reactive and phagocytic microglia (Fig. 4I). The nuclear-to-cytoplasmic Nrf2 ratio on the ipsilateral side in NDP-MSH-treated mice (2.2) was significantly higher than that in PBS-treated mice (1.6; Fig. 4J). The same treatment regimen in $M C 1 R^{e / e}$ mice did not result in differences in striatal dopamine or SN TH-positive cell counts between NDP-MSH- and PBS-treated mice (Fig. 4E, F), indicating that functioning MC1R is required for mediating the protective effects of NDP-MSH despite the broad affinity of NDP-MSH for other melanocortin receptors. 
MC1R activation alleviates $\alpha$ Syn oligomerization and neurotoxicity by activating Nrf2 in vitro

Given that MC1R genetic and pharmacological manipulations alter Nrf2 signaling, we further explored the role of Nrf2 in the protective actions of MC1R against aSyn pathologies in vitro using the human embryonic kidney cell line HEK293T. Transfection of human WT aSyn resulted in expression of human aSyn in both monomer and oligomer forms as demonstrated by Western blotting after crosslinking to stabilize aSyn oligomers (Fig. S5A). Endogenous aSyn in HEK293T cells is limited or undetectable[43]; no aSyn was detected in non-transfected or vector-transfected cells (Fig. S5A). Co-transfection with human MC1R in HEK293T cells overexpressing aSyn led to a significant increase in Nrf2 compared with non-transfected or vector-transfected cells at both the protein and mRNA levels as determined by Western blotting and qPCR (Fig. 5A, B). The human MC1R, similar to the mouse receptor, has been shown to have significant ligand-independent signaling when overexpressing in HEK293 cells[23]. Fluorescence staining showed an increased ratio of nuclear-to-cytoplasmic Nrf2 (Fig. 5C). In addition, qPCR showed increased HO-1 mRNA expression in MC1R and aSyn co-transfected cells (Fig. 5D). These results suggest that Nrf2 is induced and activated by MC1R overexpression in HEK293T cells overexpressing aSyn. Immunoblotting for human aSyn showed significantly reduced aSyn in both its monomer form and higher molecular weight species in cells overexpressing MC1R compared with vector-transfected cells (Fig. 5E, F).

We next employed shNrf2 to knock down Nrf2 in HEK293T cells transfected with aSyn and MC1R or control vector. Transduction of shNrf2 or control scRNA were achieved using lentivirus, and overexpression of MC1R and knock down of Nrf2 were confirmed by Western blotting (Fig. 5F-H). The reduction in aSyn monomer and oligomers by MC1R overexpression was reversed in cells transduced with shNrf2 as assessed by immunoblotting. Quantification revealed no difference in aSyn monomer or oligomers in vector-transfected cells treated with scRNA (Fig. 5F, I). These results suggest that Nrf2 has an essential role in mediating the influence of MC1R on aSyn in HEK293T cells. 
To further confirm Nrf2 as a downstream effector of MC1R neuroprotection, we prepared cortical primary neurons from WT and $M C 1 R^{\mathrm{e} / \mathrm{e}}$ mouse embryos and performed MAP2 and GFAP immunofluorescence staining. A high purity of neurons in the culture was supported by abundant MAP2-postive cells and rare GFAP-stained cells (Fig. S5B). Endogenous MC1R expression in cortical neurons was detected by immunohistochemistry (Fig. 5J). Previous studies reported that $M C 1 R$ is expressed in CNS neurons in late gestation[44] and neuron cultures prepared from C57BL/6J embryos[18]. To characterize aSyn toxicity and the effect of MC1R in primary cortical neurons, cell preparations from WT and $M C 1 R^{e / e}$ mice were transduced with BiFC aSyn AAV or control venus AAV at day in vitro (DIV)3 and treated with the MC1R agonist NDP-MSH 2 days later. Transduction of venus AAV or aSyn oligomerization and morphological changes were visualized by fluorescence at DIV9. Similar to our previous report in mice[24], oligomeric BiFC aSyn was associated with beaded and punctate structures in WT neurons (Fig. S5C). Consistently, higher cytotoxicity was indicated by a LDH release assay in BiFC aSyn-transduced cells compared with venus-transduced cells at DIV12. Treatment with NDP-MSH attenuated aSyn cytotoxicity. aSyn oligomerization and the related appearance of distorted and segmented neurites were more evident in $M C 1 R^{e / e}$ neurons (Fig. S5C). NDP-MSH treatment of $M C 1 R^{e / e}$ neurons did not alter aSyn cytotoxicity, suggesting that the protective effect of NDP-MSH against $\alpha$ Syn depends on functioning MC1R (Fig. S5C).

We next knocked down Nrf2 in WT primary neurons transfected with aSyn using lentiviral shNrf2. The knock-down efficiency of shNrf2 was confirmed by qPCR (Fig. 5K). Cells were also treated with NDP-MSH for 7 days, which significantly decreased LDH cytotoxicity at DIV12. There was no difference in LDH release between NDP-MSH- and vehicle-treated cells transduced with shNrf2, suggesting that shNrf2 blocked the NDP-MSH effect (Fig. 5L). The requirement of Nrf2 in the protective effect of NDP-MSH against aSyn toxicity was further demonstrated by MAP2-positive cell counting using immunohistochemistry (Fig. 5M, N). The number of surviving neurons after aSyn transfection in the shNrf2+NDP-MSH group was significantly lower than that in the scRNA+NDPMSH group. There was no significant difference between shNrf2+NDP-MSH and shNrf2+PBS groups (Fig. 5M, N). Treatment with shNrf2 significantly increased LDH and tended to decrease 
the number of MAP2-positive cells as compared with scRNA in cortical primary neurons (Fig. 5L, $\mathrm{M}, \mathrm{N}$ ), consistent with the previously reported role of endogenous Nrf2 in maintaining neuronal cell survival[4,5,45].

\section{MC1R is present in dopaminergic neurons in the human SN but reduced in PD patients}

We next determined whether MC1R is expressed in the $\mathrm{SN}$ in individuals with no neurological conditions and whether it is altered in patients with PD. Immunohistochemistry showed distinct positive staining for MC1R in the $\mathrm{SN}$, where $\mathrm{TH}$-positive dopaminergic neurons were abundant (Fig. 6A). Examination of cellular morphology revealed that MC1R was expressed on the membrane and in the cytoplasm, similar to our previous report in mice[20], and cell projections were also distinctly stained. Omitting primary or secondary antibodies yielded no specific staining, with only dark pigment then visible. Fluorescence staining for MC1R, TH, and nuclear DAPI showed that most TH-positive cells stained positive for MC1R, indicating the presence of $M C 1 R$ in dopaminergic neurons (Fig. 6B). Fluorescence double-staining showed almost no overlap of MC1R and GFAP (Fig. 6B), consistent with our findings in mice (Fig. S4B).

We next examined postmortem SN tissue from patients with PD. Peroxidase immunostaining indicated fewer MC1R-positive cells in the SN from PD patients compared with controls (Fig. 6C). Loss of TH-positive cells was also evident in the SN of PD patients. After fluorescence doublestaining, there appeared to be fewer cells in the SNpc stained with both MC1R and TH compared with controls (Fig. 6C, D). Western blotting using SN tissue showed a loss of MC1R in proportion to a loss of TH in PD patients compared with controls (Fig. 6E). These observations were overall consistent across all 5 PD patients and 4 controls.

\section{Discussion}

We previously reported protective effects of melanoma-related MC1R in nigrostriatal dopaminergic neuron survival under basal conditions and in toxin models of PD[20]. In the present study, we found exacerbated aSyn pathology following AAV-mediated overexpression of aSyn in the SN of $M C 1 R^{\mathrm{e} / \mathrm{e}}$ mice carrying a loss-of-function mutation. Increased oligomeric $\alpha \mathrm{Syn}$, especially amyloid- 
like aSyn fibrils and p-aSyn, indicated pathologic aSyn aggregation in $M C 1 R^{e / e}$ mice. Existing evidence connecting MC1R and aSyn is scarce and indirect. In vitro studies using melanoma cells, which express MC1R, indicates that aSyn is highly expressed and promotes melanoma cell survival[ $[46,47]$. In addition, aSyn is shown to reduce UV-induced melanin synthesis in melanoma cells, suggesting a possible inhibitory effect of aSyn on the melanin pathway that is controlled by $\operatorname{MC1R}[48]$

As a consequence of exacerbated aSyn pathology, $M C 1 R^{\mathrm{e} / \mathrm{e}}$ mice with $\mathrm{SN}$-targeted overexpression of aSyn displayed exacerbated dopaminergic deficits anatomically and neurochemically. This exacerbation cannot be solely explained by a preexisting dopaminergic dysfunction previously demonstrated in these mice around the same age[20]. The ability of human $M C 1 R$ to rescue the dopamine deficits indicates the specificity of this MC1R effect. The human MC1R transgene, under its human physiological promoter, expresses less but more potent MC1R than the mouse gene and restores pigmentation in $M C 1 R^{\mathrm{e} / \mathrm{e}}$ mice[23]. Although our results suggest that the $\mathrm{SN}$ expression pattern of the transgenic human $M C 1 R$ was similar to the endogenous WT mouse $M C 1 R$ and exerted a protective effect, they do not indicate whether the rescue was partial or complete or whether the transgene might have had trophic effects, as no Tg and WT littermates were compared. Collectively, our findings of $M C 1 R$ disruption-induced impairment of aSyn defense and human $M C 1 R$-mediated rescue strongly support MC1R-specific dopaminergic protection against aSyn.

The ability and specificity of MC1R to protect against aSyn-induced dopaminergic deficits were further demonstrated by BMS-470539, a selective MC1R agonist with modest blood-brain barrier permeability, and by locally delivered NDP-MSH, a broader agonist for melanocortin receptors with no brain penetrance, in WT but not MC1R mutant mice. Commercially known as Scenesse ${ }^{\circledR}$, NDPMSH is pending U.S. Food and Drug Administration review after being approved by the European Medicines Agency to treat patients with erythropoietic protoporphyria. The neuroprotective effects of systemically adminstered NDP-MSH have been reported in models of ischemic stroke, traumatic brain injury, spinal cord injury, and Alzheimer's disease[15-17]. A more recent study demonstrates the MC1R-dependent neuroprotective effects of intravenously injected NDP-MSH in mouse models 
of neuroinflammatory disease involving a compromised blood-brain barrier[18]. Activation of MC1R in the brain locally as well as peripherally, perhaps through improvements in the systemic environment, could theoretically protect the nigrostriatal system and may not necessarily exclude each other. While the involvement of peripheral MC1R cannot be ruled out, especially in the context of BMS-470539 neuroprotection, the efficacy of locally administered NDP-MSH suggests that CNS MCIR has protective functions, which is strongly supported by the similar protective effects observed in primary neuronal cultures. Therefore, our study reveals that neuronal MC1R functions as a protective signaling inducer against aSyn neurotoxicity. Further differentiating peripheral versus CNS MC1R actions using cell- or tissue-specific knock-out (e.g., in tyrosinase- and/or THexpressing cells) in addition to global $M C 1 R^{\mathrm{e} / \mathrm{e}}$ would be important for further target validation and drug development and for gaining a better understanding of the mechanisms underlying MC1Rmediated dopaminergic defense.

aSyn, especially in its oligomeric form, induces neuroinflammation and oxidative stress, which may contribute to neurodegeneration in PD[43,49]. We found that exacerbation and protection of aSyn dopaminergic neurotoxicity by genetic and pharmacological MC1R manipulations were accompanied by altered pro-inflammatory cytokines and microglia activation status in the SN. Immune cells including microphages, monocytes, and endothelial cells express MC1R[12,13]. Expression of MC1R has been reported in a human microglial cell line[50] but not in rat primary microglia[51]. We could not detect MC1R in microglial cells by immunofluorescence doublestaining, suggesting that microglial responses may be mediated by neuronal MC1Rs and alterations in neuronal activities. Together with the reported MC1R-dependent protective effect of NDP-MSH against neuroinflammation[18], our findings further support the broad role of MC1R in immunomodulation and inflammation, not only in the periphery[13] but also in the CNS. Despite the previously reported expression of MC1R in a human astrocyte cell line and suggested glial cell MC1R-mediated inhibition of TNF- $\alpha$ by $\alpha-\mathrm{MSH}$ in a mouse model of brain inflammation[50,52,53], our double-labeling showed rare colocalization of MC1R and GFAP. MC1R manipulation was not associated with the gliosis that we and others have observed following aSyn overexpression, at least at the time points analyzed. 
Additionally, given the predominant transduction of aSyn and MC1R expression in neurons, the altered inflammation and oxidative damage in the ventral midbrain were likely effects of altered Nrf2 signaling in neurons in response to MC1R manipulation. Nrf2, known as a master regulator of the immune system and oxidative stress, was shown to be regulated by MC1R, with impaired induction and activation following aSyn expression in $M C 1 R^{\mathrm{e} / \mathrm{e}}$ mice; and conversely, with enhanced induction and activation following genetic and pharmacological activation of MC1R in vivo. In cellular models of aSyn pathologies, including primary neuron cultures, the role of Nrf2 was indispensable for the counteractions of MC1R genetic and pharmacological activation. CAMP response element-binding protein, which is responsive to MC1R, has been identified within Nrf2 promoter regions, and NDP-MSH activates Nrf2 in human skin[54]. Our results directly showed that Nrf2 is a downstream mediator of the protective effects of MC1R activation. These findings also support the ability of Nrf2 in neurons to be responsive[45], both in vivo and in vitro, to MC1R manipulation in this context. As Nrf2 may be altered in Parkinson's and related neurodegenerative diseases, and Nrf2 activators have progressed to active clinical practice or development as neurotherapeutics[5,45,55,56], our results provide not only mechanistic insight into MC1R defense against $\alpha$ Syn pathologies but also therapeutic implications for the possible use of Nrf2 activators in PD patients, especially those carrying partial or complete loss-of-function MC1R variants.

The translational significance of our findings is further highlighted by the presence of MC1R in dopaminergic neurons in the human $\mathrm{SN}$ and the suggestion of reduced MC1R expression in PD patients vs. controls. MC1R immunoreactivity in periaqueductal gray neurons is reported in fixed human brain sections[57]. Mykicki et al. described the expression of MC1R in human neuronal cells differentiated from a progenitor cell line and in neurons from postmortem human brain tissues[18]. The same study reported reduced brain MC1R expression in multiple sclerosis patients, although no brain regions or neuron types were specified[18]. The remarkable overlap of MC1R and TH in the $\mathrm{SN}$ observed in our study suggests primary, if not exclusive, dopaminergic neuronal functions of MC1R within the SN. Although it remains to be determined whether loss of MC1R in PD patients precedes loss of dopaminergic neurons, the evidence presented by this and other studies supports that MC1R is involved mechanistically in the pathophysiology of PD and is a promising therapeutic 
target for PD and related disorders. The findings encourage development of CNS-penetrant MC1R agonists as well as potential repurposing of existing, primarily peripherally acting MC1R agonists, as candidate disease-modifying therapy for PD. Given the links among MC1R loss of function, red hair, melanoma, and PD, our findings also provide evidence of a possible MC1R basis for the wellestablished link between PD and melanoma. The similar protection observed in vitro in cells that are considered non-pigmented and lack pigmentation machinery, including HEK293T cells and primary cortical neurons, supports non-pigmentary pathways of MC1R actions. Further studies are needed to determine whether $M C 1 R$ as the key pigmentation gene acts through pigmentation and whether neuromelanin or melanin in the periphery is involved in MC1R neuroprotection in vivo.

\section{Conclusions}

By using genetic and pharmacological approaches, our study demonstrated in multiple in vivo and in vitro models that MC1R loss-of-function leads to exacerbated PD-associated aSyn pathologies. Conversely MC1R activation is neuroprotective against aSyn-induced neurotoxicity. The protective effects of MC1R can be mediated by Nrf2, at least in vitro. Together with the human postmortem tissue data and the previous epidemiological and biological studies from our group and others', these results suggest that MC1R signaling plays a role in neurodegeneration in PD. They provide evidence for MC1R as a therapeutic target and a rationale for development of MC1R-activating strategies for PD.

\section{List of abbreviations}

$\begin{array}{ll}\text { AAV } & \text { adeno-associated virus } \\ \text { BiFC } & \text { bimolecular fluorescence complementation } \\ \text { GCLC } & \text { glutamate-cysteine ligase subunit C } \\ \text { GFAP } & \text { glial fibrillary acidic protein } \\ \text { HO1 } & \text { heme oxygenase 1 } \\ \text { HPLC } & \text { high-performance liquid chromatography } \\ \text { iba1 } & \text { ionized calcium binding adapter molecule 1 }\end{array}$




\begin{tabular}{ll} 
ICAM1 & intercellular adhesion molecule 1 \\
IL-6 & interleukin (IL)-6 \\
LDH & lactate dehydrogenase \\
MC1R & Melanocortin 1 receptor \\
NDP-MSH & Nle4,D-Phe7- $\alpha$-MSH \\
NQO1 & NAD(P)H quinone dehydrogenase 1 \\
Nrf2 & nuclear factor erythroid 2-related factor 2 \\
PD & Parkinson disease \\
p-aSyn & phosphorylated aSyn at serine 129 \\
qPCR & quantitative polymerase chain reaction \\
ScRNA & scrambled RNA \\
SN & substantia nigra \\
SNpc & SN pars compacta \\
Tg & transgenic \\
TH & tyrosine hydroxylase \\
TNF & tumor necrosis factor \\
WT & wild-type \\
$\alpha-M S H$ & alpha-melanocyte stimulating hormone \\
aSyn & alpha-synuclein \\
\hline
\end{tabular}

Declarations

\section{Ethics approval and consent to participate}

All animal protocols were approved by the Massachusetts General Hospital Animal Care and Use Committee (2018N000039).

Human postmortem tissue study was approved by the Partners Human Research Committee (2018P000367).

\section{Consent for publication}

Not applicable

\section{Availability of data and materials}


All data generated or analyzed during this study are included in this published article and its supplementary information files.

\section{Competing interests}

The authors declare that they have no competing interests.

\section{Funding}

This work was supported by NIH grants R01NS102735 and R01NS110879, the Michael J. Fox Foundation and the Aligning Science Across Parkinson's Initiative ASAP-000312, the Milstein Medical Asian American Partnership Foundation 2015, and the Farmer Family Foundation Initiative for Parkinson's Disease Research.

\section{Authors' contributions}

WC performed most of the experiments; DF assisted with virus injection and performed HPLC analysis; YL performed HPLC analysis; CRV assisted with immunohistochemistry in human tissues; YX was responsible for animal husbandry, breeding, and genotyping; PM provided viral vectors and provided guidance on their use and data interpretation; MF reviewed human tissue results and provided guidance on interpretation of the results; DEF provided $M C 1 R^{\mathrm{e} / \mathrm{e}}$ mice and guidance on experiment design and data interpretation; MAS provided guidance on the project direction and data interpretation; XC designed and supervised the study; WC and XC wrote the paper with critical edits from DEF and MAS. All authors read and approved the final manuscript.

\section{Acknowledgments}

We thank Professor lan J. Jackson (University of Edinburgh) for providing $M C 1 R^{\mathrm{e} / \mathrm{T}} \mathrm{Tg}$ mice and guidance on their use.

\section{References}

1. Polymeropoulos MH, Lavedan C, Leroy E, Ide SE, Dehejia A, Dutra A, et al. Mutation in the asynuclein gene identified in families with Parkinson's disease. Science. 1997;276:2045-7.

2. Blauwendraat $C$, Nalls MA, Singleton $A B$. The genetic architecture of Parkinson's disease. Lancet Neurol. 2020;19:170-8.

3. Abeliovich A, Gitler AD. Defects in trafficking bridge Parkinson's disease pathology and genetics. Nature. 2016;539:207.

4. Lastres-Becker I, Ulusoy A, Innamorato NG, Sahin G, Rábano A, Kirik D, et al. a-synuclein 
expression and Nrf2 deficiency cooperate to aggravate protein aggregation, neuronal death and inflammation in early-stage Parkinson's disease. Hum Mol Genet. 2012;21:3173-92.

5. Dinkova-Kostova AT, Kostov R V., Kazantsev AG, Dinkova-Kostova AT, Kostov R V., Kazantsev AG. The role of Nrf2 signaling in counteracting neurodegenerative diseases. FEBS J. 2018;285:3576-90.

6. Price ER, Horstmann MA, Wells AG, Weilbaecher KN, Takemoto CM, Landis MW, et al. $\alpha$ melanocyte-stimulating hormone signaling regulates expression of Microphthalmia, a gene deficient in Waardenburg syndrome. J Biol Chem. 1998;273:33042-7.

7. Lin JY, Fisher DE. Melanocyte biology and skin pigmentation. Nature. 2007;445:843-50.

8. Wolf Horrell EM, Boulanger MC, D'Orazio JA. Melanocortin 1 Receptor: Structure, Function, and Regulation. Front Genet. 2016;7:95.

9. Williams $\mathrm{P}$, Olsen $\mathrm{C}$. Melanocortin 1 receptor and risk of cutaneous melanoma: A metaanalysis and estimates of population burden. Int J Cancer. 2011;129:1730-40.

10. Law MH, Medland SE, Zhu G, Yazar S, Viñuela A, Wallace L, et al. Genome-Wide Association Shows that Pigmentation Genes Play a Role in Skin Aging. J Invest Dermatol. 2017; 137:1887-94.

11. Roider EM, Fisher DE. Red hair, light skin, and UV-independent risk for melanoma development in humans. JAMA dermatology. 2016;152:751-3.

12. Salazar-Onfray F, Lopez M, Lundqvist A, Aguirre A, Escobar A, Serrano A, et al. Tissue distribution and differential expression of melanocortin 1 receptor, a malignant melanoma marker. Br J Cancer. 2002;87:414.

13. Catania A, Gatti S, Colombo G, Lipton JM. Targeting melanocortin receptors as a novel strategy to control inflammation. Pharmacol Rev. 2004;56:1-29.

14. Langendonk JG, Balwani M, Anderson KE, Bonkovsky HL, Anstey A V, Bissell DM, et al. Afamelanotide for erythropoietic protoporphyria. N Engl J Med. 2015;373:48-59.

15. Schaible E-V, Steinsträßer A, Jahn-Eimermacher A, Luh C, Sebastiani A, Kornes F, et al. Single administration of tripeptide $\alpha-\mathrm{MSH}(11-13)$ attenuates brain damage by reduced inflammation and apoptosis after experimental traumatic brain injury in mice. PLoS One. 2013;8:e71056.

16. Giuliani D, Bitto A, Galantucci M, Zaffe D, Ottani A, Irrera N, et al. Melanocortins protect against progression of Alzheimer's disease in triple-transgenic mice by targeting multiple pathophysiological pathways. Neurobiol Aging. 2014;35:537-47.

17. Leone S, Noera G, Bertolini A. Melanocortins as innovative drugs for ischemic diseases and neurodegenerative disorders: established data and perspectives. Curr Med Chem. 2013;20:73550.

18. Mykicki N, Herrmann AM, Schwab N, Deenen R, Sparwasser T, Limmer A, et al. Melanocortin-1 receptor activation is neuroprotective in mouse models of neuroinflammatory disease. Sci Transl Med. 2016;8:362ra146.

19. Chen X, Feng D, Schwarzschild MA, Gao X. Red hair, MC1R variants, and risk for Parkinson's disease - a meta-analysis. Ann Clin Transl Neurol. 2017;4:212-6.

20. Chen X, Chen H, Cai W, Maguire M, Ya B, Zuo F, et al. The melanoma-linked "redhead" MC1R influences dopaminergic neuron survival. Ann Neurol. 2017;81:395-406. 
21. Chen X, Burdett TC, Desjardins CA, Logan R, Cipriani S, Xu Y, et al. Disrupted and transgenic urate oxidase alter urate and dopaminergic neurodegeneration. Proc Natl Acad Sci U S A. 2013;110:300-5.

22. Mitra D, Luo X, Morgan A, Wang J, Hoang MP, Lo J, et al. An ultraviolet-radiationindependent pathway to melanoma carcinogenesis in the red hair/fair skin background. Nature. 2012;491:449-53.

23. Jackson IJ, Budd PS, Keighren M, McKie L. Humanized MC1R transgenic mice reveal human specific receptor function. Hum Mol Genet. 2007;16:2341-8.

24. Cai W, Feng D, Schwarzschild MA, McLean PJ, Chen X. Bimolecular Fluorescence Complementation of Alpha-synuclein Demonstrates its Oligomerization with Dopaminergic Phenotype in Mice. EBioMedicine. 2018;29:13-22.

25. Jiao Y, Sun Z, Lee T, Fusco FR, Kimble TD, Meade CA, et al. A simple and sensitive antigen retrieval method for free-floating and slide-mounted tissue sections. J Neurosci Methods. 1999;93:149-62.

26. Paxinos G, Franklin KBJ. The Mouse Brain in Stereotaxic Coordinates, 2nd edition. Acad. Press. 2001.

27. West MJ, Slomianka L, Gundersen HJG. Unbiased stereological estimation of the total number of neurons in the subdivisions of the rat hippocampus using the optical fractionator. Anat Rec. 1991;231:482-97.

28. Sun A, Nguyen X V, Bing G. Comparative analysis of an improved thioflavin-s stain, Gallyas silver stain, and immunohistochemistry for neurofibrillary tangle demonstration on the same sections. J Histochem Cytochem. 2002;50:463-72.

29. Bakshi R, Zhang H, Logan R, Joshi I, Xu Y, Chen X, et al. Neuroprotective effects of urate are mediated by augmenting astrocytic glutathione synthesis and release. Neurobiol Dis.

2015;82:574-9.

30. Giulietti A, Overbergh L, Valckx D, Decallonne B, Bouillon R, Mathieu C. An overview of realtime quantitative PCR: applications to quantify cytokine gene expression. Methods. 2001;25:386401.

31. Xiao D, Bastia E, Xu Y-H, Benn CL, Cha J-HJ, Peterson TS, et al. Forebrain Adenosine A2A Receptors Contribute to L-3,4-Dihydroxyphenylalanine-Induced Dyskinesia in Hemiparkinsonian Mice. J Neurosci. 2006;26:13548-55.

32. Mazzulli JR, Armakola M, Dumoulin M, Parastatidis I, Ischiropoulos H. Cellular oligomerization of $\alpha$-synuclein is determined by the interaction of oxidized catechols with a Cterminal sequence. J Biol Chem. 2007;282:31621-30.

33. Fauvet B, Mbefo MK, Fares M-B, Desobry C, Michael S, Ardah MT, et al. a-Synuclein in central nervous system and from erythrocytes, mammalian cells, and Escherichia coli exists predominantly as disordered monomer. J Biol Chem. 2012;287:15345-64.

34. Cipriani S, Desjardins CA, Burdett TC, Xu Y, Xu K, Schwarzschild MA. Urate and its transgenic depletion modulate neuronal vulnerability in a cellular model of Parkinson's disease. PLoS One. 2012;7:e37331.

35. Hilgenberg LGW, Smith MA. Preparation of dissociated mouse cortical neuron cultures. J Vis Exp. 2007;10:e562.

36. Royo NC, Vandenberghe LH, Ma J-Y, Hauspurg A, Yu L, Maronski M, et al. Specific AAV 
serotypes stably transduce primary hippocampal and cortical cultures with high efficiency and low toxicity. Brain Res. 2008;1190:15-22.

37. McFarland NR, Lee J, Hyman BT, McLean PJ. Comparison of transduction efficiency of recombinant AAV serotypes 1, 2, 5, and 8 in the rat nigrostriatal system. J Neurochem. 2009;109:838-45.

38. Klein RL, Dayton RD, Leidenheimer NJ, Jansen K, Golde TE, Zweig RM. Efficient neuronal gene transfer with AAV8 leads to neurotoxic levels of tau or green fluorescent proteins. Mol Ther. 2006;13:517-27.

39. Anderson JP, Walker DE, Goldstein JM, De Laat R, Banducci K, Caccavello RJ, et al. Phosphorylation of Ser-129 is the dominant pathological modification of $\alpha$-synuclein in familial and sporadic lewy body disease. J Biol Chem. 2006;281:29739-52.

40. Karampetsou M, Ardah MT, Semitekolou M, Polissidis A, Samiotaki M, Kalomoiri M, et al. Phosphorylated exogenous alpha-synuclein fibrils exacerbate pathology and induce neuronal dysfunction in mice. Sci Rep. 2017;7:16533.

41. Quinti L, Naidu SD, Träger U, Chen X, Kegel-Gleason K, Llères D, et al. KEAP1-modifying small molecule reveals muted NRF2 signaling responses in neural stem cells from Huntington's disease patients. Proc Natl Acad Sci U S A. 2017;114:E4676-85.

42. Chen P-C, Vargas MR, Pani AK, Smeyne RJ, Johnson DA, Kan YW, et al. Nrf2-mediated neuroprotection in the MPTP mouse model of Parkinson's disease: Critical role for the astrocyte. Proc Natl Acad Sci. 2009;106:2933-8.

43. Delenclos M, Burgess JD, Lamprokostopoulou A, Outeiro TF, Vekrellis K, McLean PJ. Cellular models of alpha-synuclein toxicity and aggregation. J Neurochem. 2019;150:566-76.

44. Thomas AC, Heux P, Santos C, Arulvasan W, Solanky N, Carey ME, et al. Widespread dynamic and pleiotropic expression of the melanocortin-1-receptor (MC1R) system is conserved across chick, mouse and human embryonic development. Birth Defects Res. 2018;110:443-55.

45. Liddell J. Are astrocytes the predominant cell type for activation of Nrf2 in aging and neurodegeneration? Antioxidants. 2017;6:65.

46. Rodriguez-Leyva I, Chi-Ahumada E, Mejía M, Castanedo-Cazares JP, Eng W, Saikaly SK, et al. The Presence of Alpha-Synuclein in Skin from Melanoma and Patients with Parkinson's Disease. Mov Disord Clin Pract. 2017;4:724-32.

47. Turriani E, Lázaro DF, Ryazanov S, Leonov A, Giese A, Schön M, et al. Treatment with diphenyl-pyrazole compound anle138b/c reveals that $\alpha$-synuclein protects melanoma cells from autophagic cell death. Proc Natl Acad Sci. 2017;114:E4971-7.

48. Pan T, Zhu J, Hwu W-J, Jankovic J. The role of alpha-synuclein in melanin synthesis in melanoma and dopaminergic neuronal cells. PLoS One. 2012;7:e45183.

49. Alam P, Bousset L, Melki R, Otzen DE. a-synuclein oligomers and fibrils: a spectrum of species, a spectrum of toxicities. J Neurochem. 2019;150:522-34.

50. Lindberg C, Hjorth E, Post C, Winblad B, Schultzberg M. Cytokine production by a human microglial cell line: Effects of ßamyloid and $\alpha$-melanocyte-stimulating hormone. Neurotox Res. 2005;8:267-76.

51. Carniglia L, Durand D, Caruso C, Lasaga M. Effect of NDP- $\alpha-M S H$ on PPAR- $y$ and- $\beta$ expression and anti-inflammatory cytokine release in rat astrocytes and microglia. PLoS One. 2013;8:e57313. 
52. Wong KY, Rajora N, Boccoli G, Catania A, Lipton JM. A potential mechanism of local antiinflammatory action of alpha-melanocyte-stimulating hormone within the brain: modulation of tumor necrosis factor-alpha production by human astrocytic cells. Neuroimmunomodulation. 1997;4:37-41.

53. Rajora N, Boccoli G, Burns D, Sharma S, Catania AP, Lipton JM. $\alpha-M S H$ modulates local and circulating tumor necrosis factor- $\alpha$ in experimental brain inflammation. J Neurosci. 1997;17:21816.

54. Kokot A, Metze D, Mouchet N, Galibert M-D, Schiller M, Luger TA, et al. Alpha-melanocytestimulating hormone counteracts the suppressive effect of UVB on Nrf2 and Nrf-dependent gene expression in human skin. Endocrinology. 2009;150:3197-206.

55. Petrillo S, Schirinzi T, Di Lazzaro G, D’Amico J, Colona VL, Bertini E, et al. Systemic activation of Nrf2 pathway in Parkinson's disease. Mov Disord. 2020;35:180-4.

56. Ramsey CP, Glass CA, Montgomery MB, Lindl KA, Ritson GP, Chia LA, et al. Expression of Nrf2 in neurodegenerative diseases. J Neuropathol Exp Neurol. 2007;66:75-85.

57. Xia Y, Wikberg JE, Chhajlani V. Expression of melanocortin 1 receptor in periaqueductal gray matter. Neuroreport. 1995;6:2193-6. 
Figures
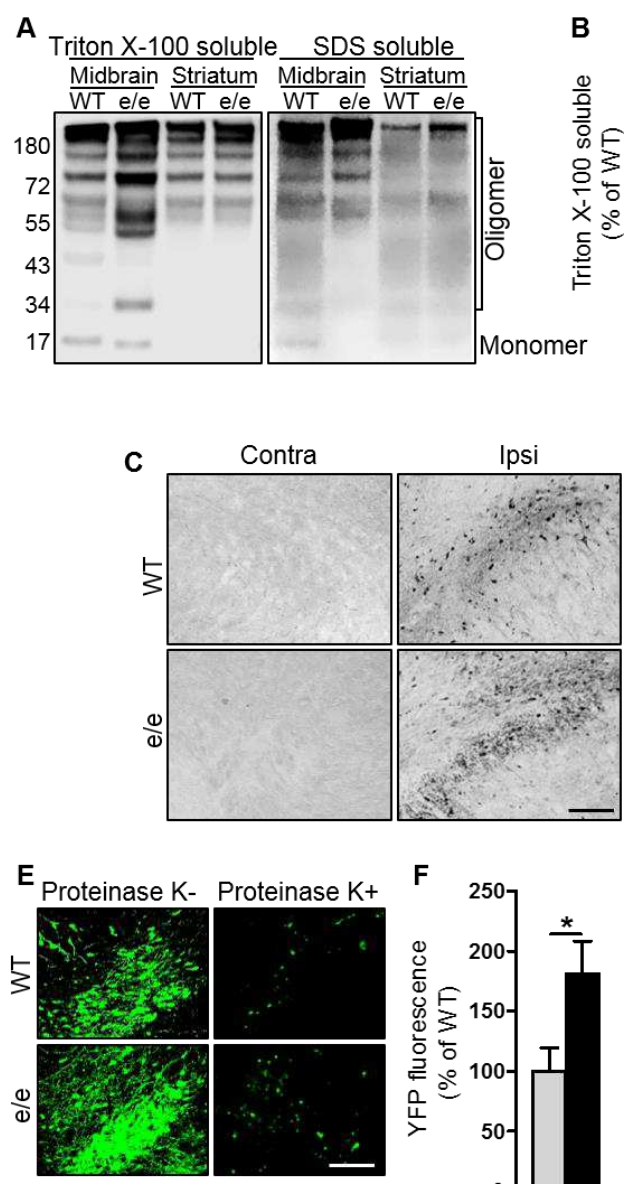
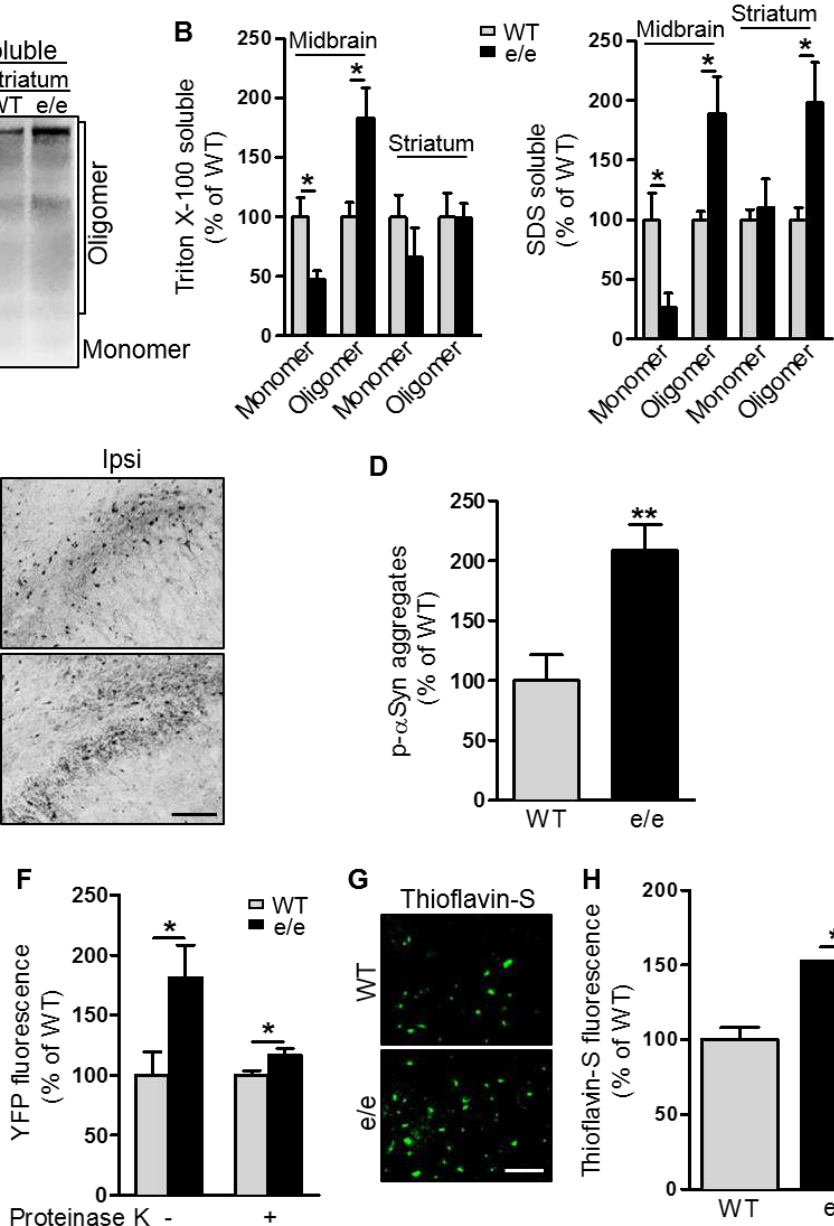

D

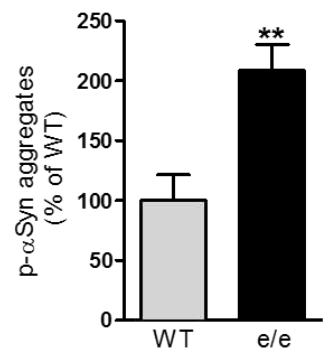

G
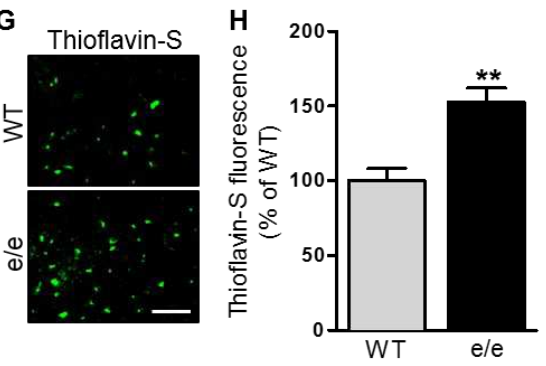

Fig. 1. MC1R disruption exacerbates synucleinopathies in the nigrostriatal pathway in an aSyn AAV mouse model. $M C 1 R^{e / e}$ and WT mice were injected unilaterally with human WT aSyn AAV into the SN and sacrificed 8 weeks later: (A) Immunoblot of human aSyn species in Triton X100 -soluble and -insoluble SDS-soluble fractions in ipsilateral ventral midbrain and striatum and (B) quantification of aSyn monomers and oligomers. $n=3$ mice/group. (C) p-aSyn staining and (D) quantification of $p$ - $\alpha$ Syn aggregates in the ipsilateral $S N . n=3$ mice/group. Scale bar, $50 \mu m$. $M C 1 R^{\mathrm{e} / \mathrm{e}}$ and WT mice were injected unilaterally with BiFC human WT aSyn AAV into the SN and sacrificed 8 weeks later: (E) VenusYFP fluorescence before and after proteinase $\mathrm{K}$ treatment and (F) quantification of venusYFP fluorescence density in the ipsilateral SN. $n=4$ mice/group. Scale bar, $50 \mu \mathrm{m}$. (G) Thioflavin-S staining and $(\mathrm{H})$ quantification of thioflavin-S fluorescence density in the ipsilateral SN. $\mathrm{n}=4$ mice/group. Scale bar, $50 \mu \mathrm{m} .{ }^{*} P<0.05,{ }^{* *} P<0.01$. 

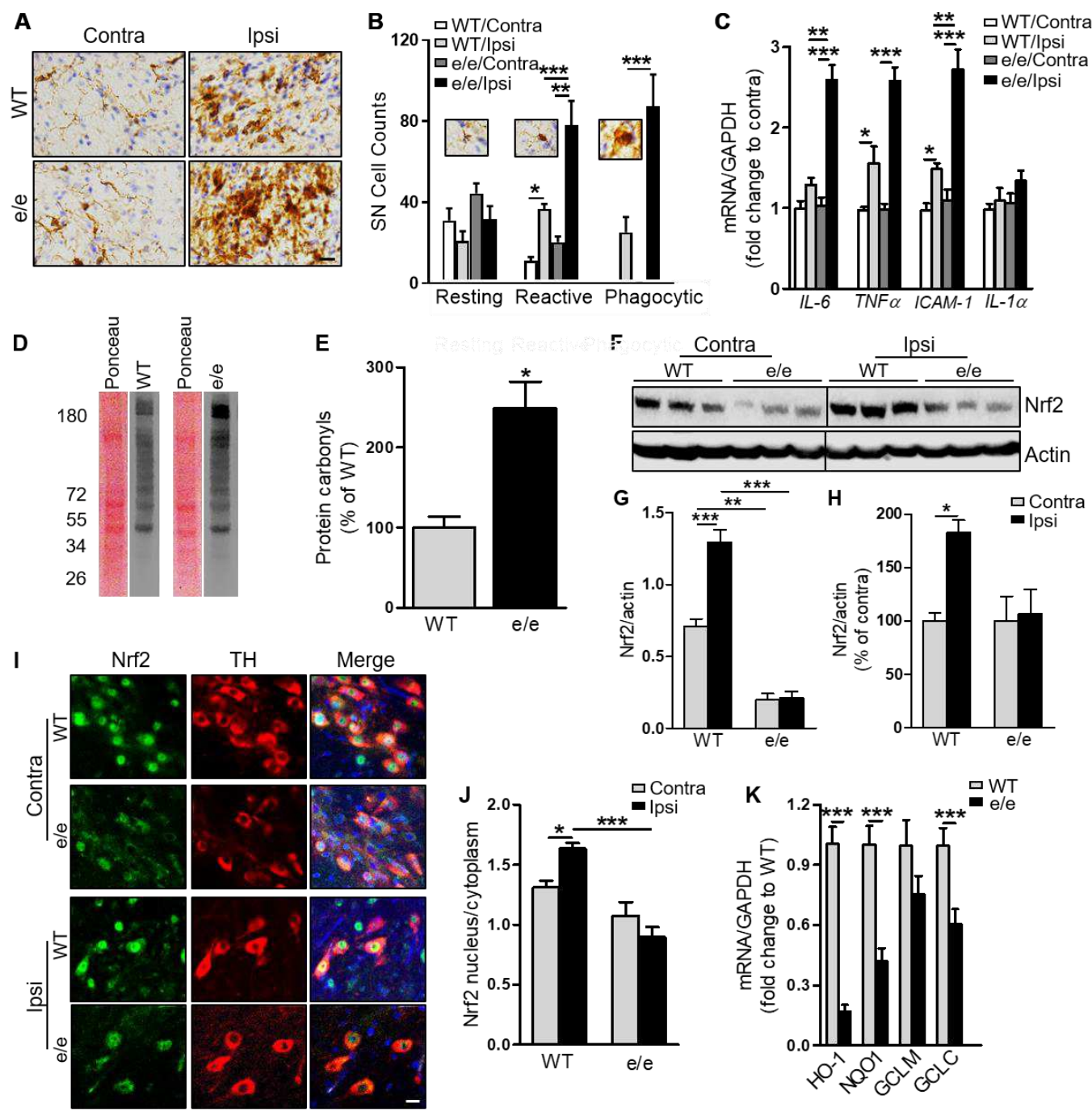

Fig. 2. MC1R disruption amplifies microglia activation and alters Nrf2 response to aSyn overexpression in the nigrostriatal pathway. $M C 1 R^{\mathrm{e} / \mathrm{e}}$ and $\mathrm{WT}$ mice were injected unilaterally with human WT aSyn AAV into the SN and sacrificed 8 weeks later: (A) lba1 staining and (B) morphological classification and quantification of iba1-positive cells in the SN. $n=4$ mice/group. Scale bar, $30 \mu \mathrm{m}$. (C) IL-1a, IL-6, TNFa, and ICAM1 mRNA levels in ventral midbrain. $\mathrm{n}=5$ mice/group. (D) Representative oxyblots for protein carbonyls and the corresponding Ponceau S staining in the ipsilateral ventral midbrain and $(E)$ quantification of band density. $n=3$ mice/group. (F) Immunoblot for Nrf2 using ventral midbrain tissue and (G) quantification of Nrf2 band density in original values or $(\mathrm{H})$ normalized to contralateral side values. $n=3$ mice/group. (I) SN sections double-stained for Nrf2 and TH and (J) quantification of nuclear and cytoplasmic Nrf2. $n=3$ 
mice/group. Scale bar, $20 \mu \mathrm{m}$. (K) mRNA levels of Nrf2 target genes HO-1, NQO1, GCLM, and GCLC in the ipsilateral ventral midbrain. $\mathrm{n}=5$ mice/group. ${ }^{\star} P<0.05,{ }^{* \star} P<0.01,{ }^{\star \star *}{ }^{*}<<0.001$.
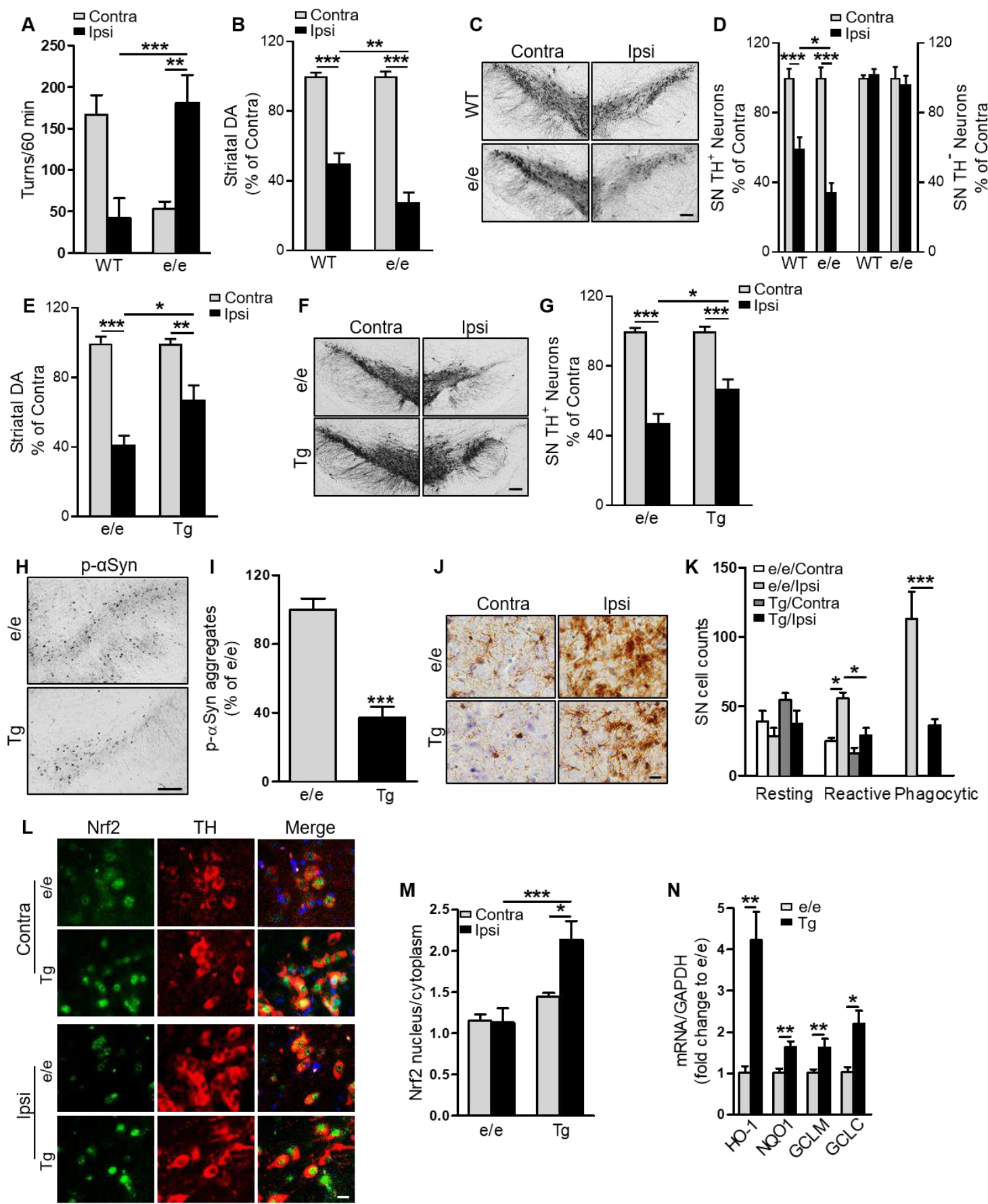

Fig. 3. aSyn-induced dopaminergic neurotoxicity is exacerbated by MC1R disruption and reversed by human $M C 1 R$ transgene. $M C 1 R^{e / e}$ and WT mice were injected unilaterally with human WT aSyn AAV into the SN. (A) Contralateral and ipsilateral turns induced by amphetamine 
12 weeks post-AAV injection. $\mathrm{n}=12$ mice/group. (B) Striatal dopamine content 16 weeks post-AAV injection. $n=12$ mice/group. (C) TH staining and (D) stereological quantification of TH-positive and -negative cells in the SN 16 weeks post-AAV injection. $n=12$ mice/group. Scale bar, $100 \mu \mathrm{m}$. $M C 1 R^{\mathrm{e} / \mathrm{e}} \mathrm{Tg}$ and $M C 1 R^{\mathrm{e} / \mathrm{e}}$ mice were injected unilaterally with human WT aSyn AAV into the SN. (E) Striatal dopamine content 16 weeks post-AAV injection. $n=6-7$ mice/group. $(F)$ TH staining and $(G)$ stereological quantification of $\mathrm{TH}$-positive and negative cells in the SN 16 weeks post-AAV injection. $n=6-7$ mice/group. Scale bar, $100 \mu \mathrm{m}$. (H) p-aSyn staining and (I) quantification of p-aSyn aggregates in the ipsilateral SNpc 12 weeks post-AAV injection. $n=4$ mice/group. Scale bar, $50 \mu \mathrm{m}$. (J) Iba1 staining and morphological classification and (K) quantification of iba1-positive cells in the SNpc 12 weeks post-AAV injection. $\mathrm{n}=4$ mice/group. Scale bar, $30 \mu \mathrm{m}$. (L) Nrf2 and TH doublelabeling and (M) quantification of nuclear and cytoplasmic Nrf2 in the SNpc 12 weeks post-AAV injection. $\mathrm{n}=4$ mice/group. Scale bar, $20 \mu \mathrm{m}$. (N) mRNA levels of Nrf2 target genes HO-1, NQO1, GCLC, and GCLM in the ipsilateral ventral midbrain 12 weeks post-AAV injection. $n=5$ mice/group. ${ }^{\star} P<0.05,{ }^{* *} P<0.01,{ }^{* *} P<0.001$. Values in $\mathrm{B}, \mathrm{D}, \mathrm{E}, \mathrm{G}$ are presented as percentages of their own contralateral mean values. 

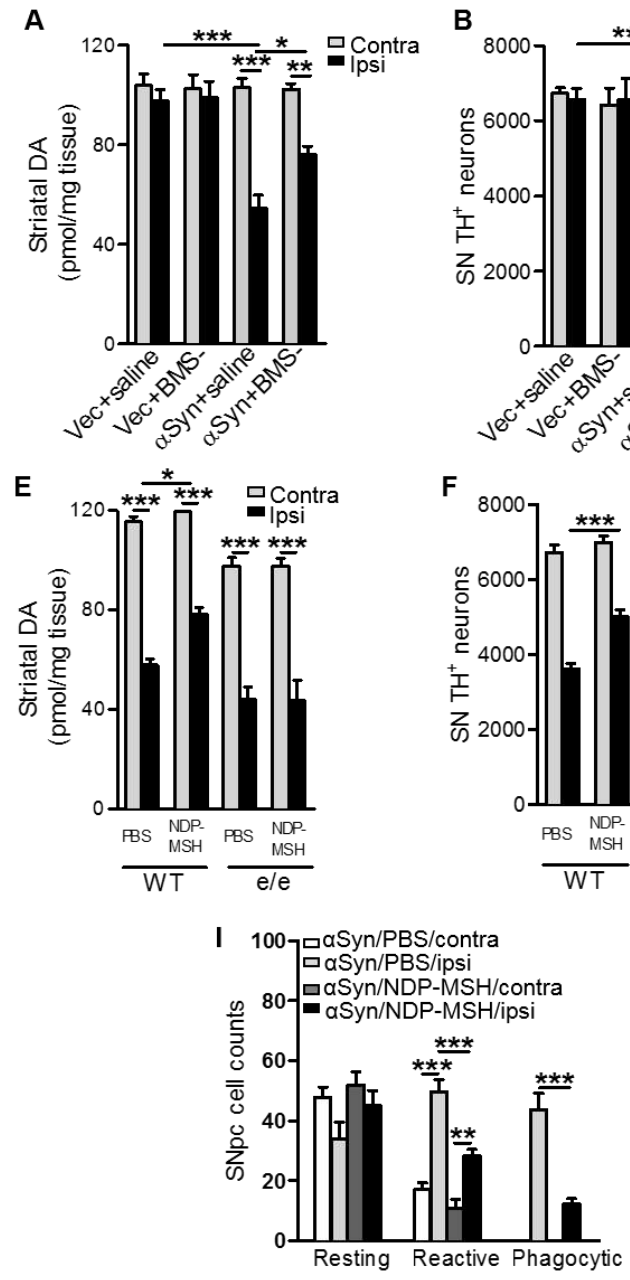
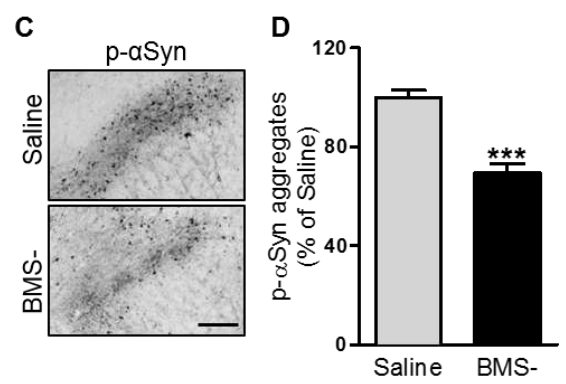

G
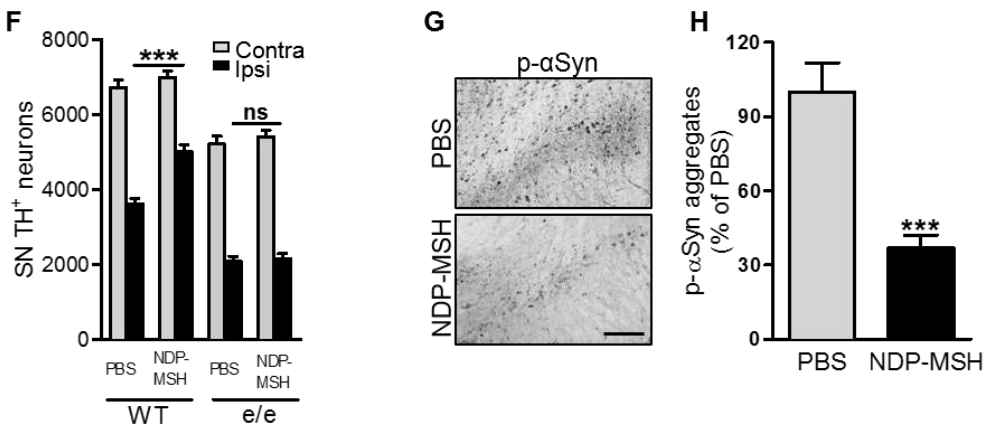

Fig. 4. MC1R agonists protect against $\alpha$ Syn-induced dopaminergic neurotoxicity. C57BI/6J mice were injected with aSyn or empty vector $(\mathrm{Vec}) \mathrm{AAV}$, treated subcutaneously with $20 \mathrm{mg} / \mathrm{kg}$ BMS-470539 (BMS-) or saline for 4 weeks, and sacrificed 16 weeks post-AAV injection. (A) Striatal dopamine content. $n=6-8$ mice/group. (B) Stereological quantification of TH-positive cells in the SN. $n=6-8$ mice/group. (C) p- $\alpha$ Syn staining and (D) quantification of $p$ - $\alpha$ Syn aggregates in the ipsilateral SN. $\mathrm{n}=4$ mice/group. Scale bar, $50 \mu \mathrm{m}$. WT and $M C 1 R^{\mathrm{e} / \mathrm{e}}$ mice were injected unilaterally with $3 \mathrm{nmol}$ NDP-MSH into the striatum and then with aSyn AAV into the SN. Mice were sacrificed 16 weeks post-AAV injection. (E) Striatal dopamine content. $n=7$ mice/group. (F) Stereological quantification of TH-positive cells in the SN. n=7 mice/group. (G) p-aSyn staining and $(H)$ quantification of $p$ - $\alpha$ Syn aggregates in the ipsilateral SN. $n=4$ mice/group. Scale bar, $50 \mu \mathrm{m}$. (I) Morphological classification 
and quantification of iba1-positive cells in the $S N$ in WT mice. $n=4$ mice/group. (J) Nuclear and cytoplasmic Nrf2 ratio in the SN in WT mice. $\mathrm{n}=4$ mice/group. ${ }^{*} P<0.05,{ }^{* \star} P<0.01,{ }^{* \star} P<0.001$. 

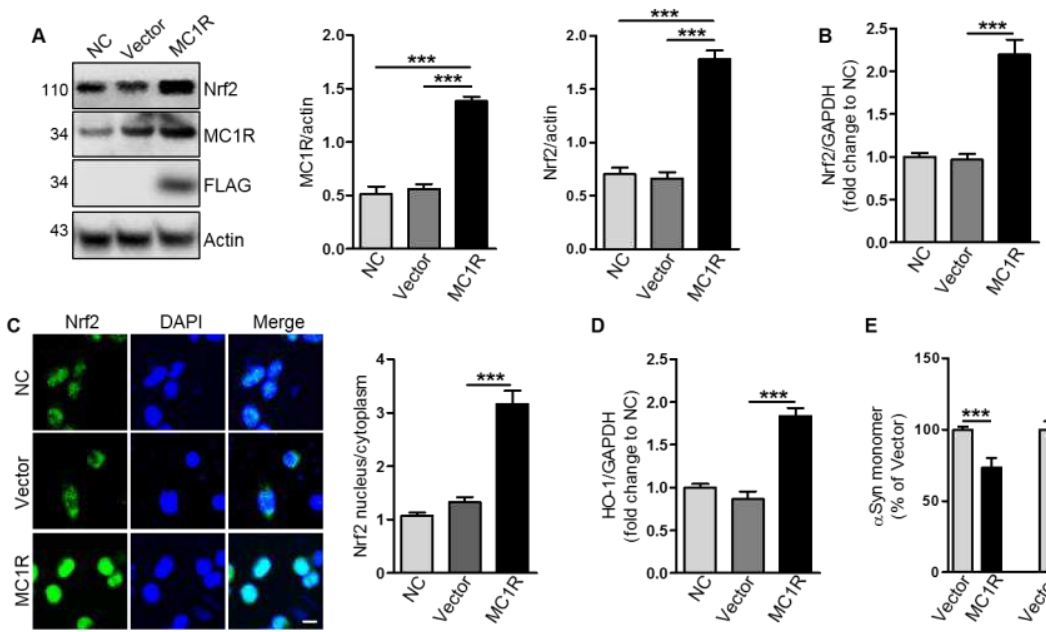

D

$\mathrm{E}$
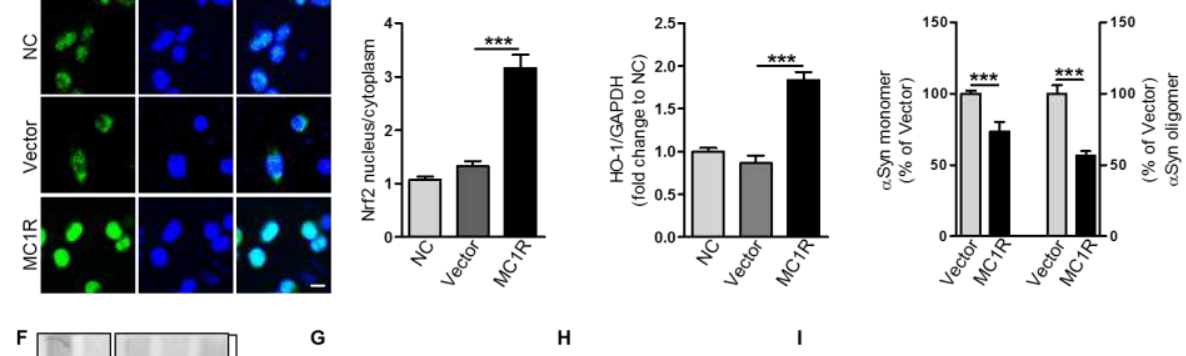

H

$$
\text { I }
$$
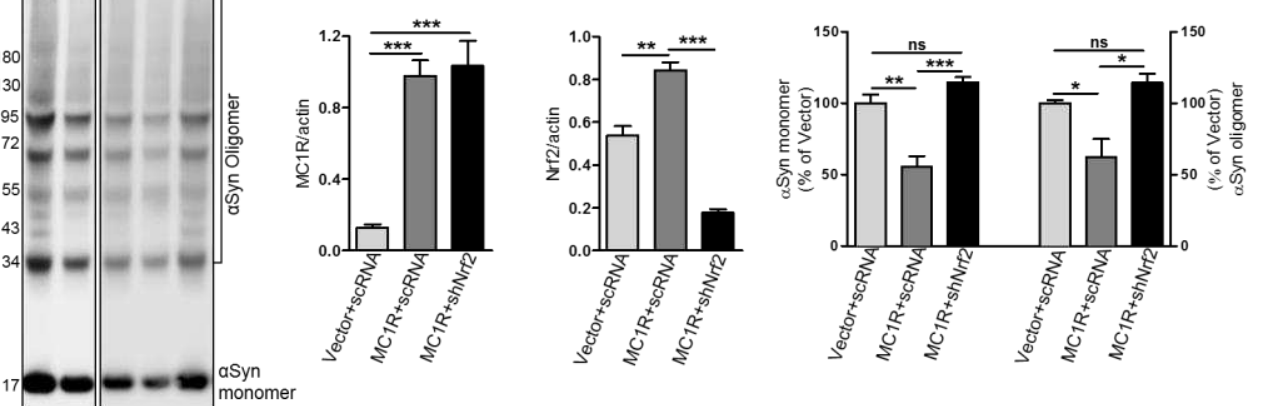

$110-\infty-2-\infty$ Nrf2

$34=-\quad-\quad-\quad$ MC1R

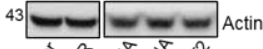

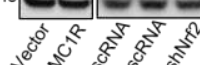

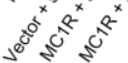
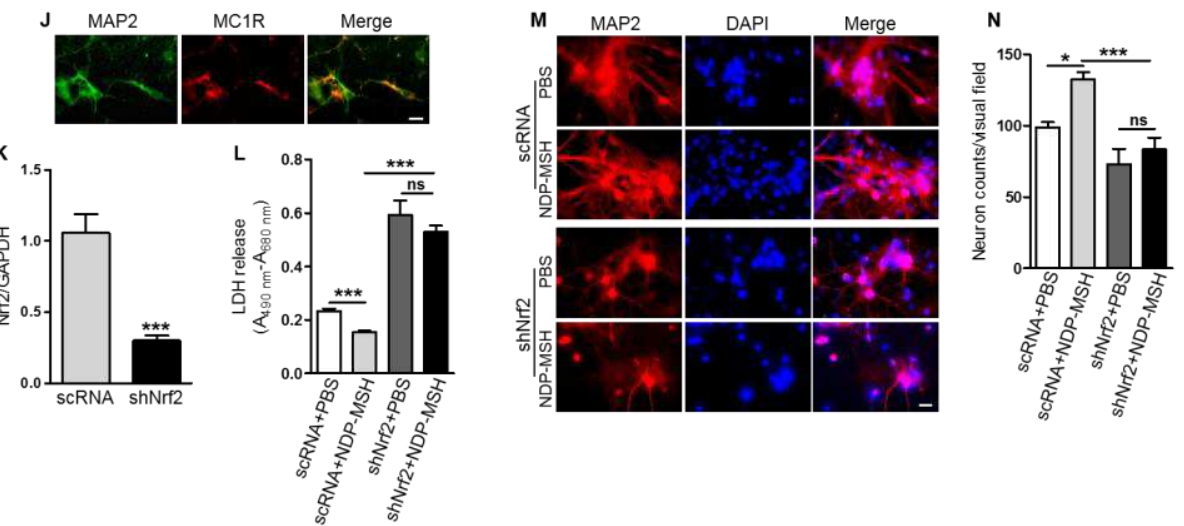

Fig. 5. MC1R activation alleviates $\alpha$ Syn oligomerization and neurotoxicity by activating Nrf2 in vitro. HEK293T cells were co-transfected with aSyn and MC1R or vector (GPRC5A-Tango). Non-transfected cells served as controls (NC): (A) Immunoblot and quantification of MC1R and Nrf2. (B) Nrf2 mRNA levels. (C) Nrf2 staining and quantification of nuclear and cytoplasmic Nrf2. 
Nuclei were stained with DAPI. Scale bar, $10 \mu \mathrm{m}$. (D) Nrf2 target gene HO-1 mRNA levels. (E and F) Immunoblot and quantification of aSyn species. HEK293T cells were transfected with aSyn, MC1R or vector control, and shNrf2 RNA or scRNA control: $(F)$ Immunoblot and ( $G$ and $H)$ quantification of MC1R and Nrf2. (F) Immunoblot and (I) quantification of aSyn species. Primary cortical neurons were prepared from embryonic day 16-17 WT mice: (J) MAP2 and MC1R staining at DIV5. Scale bar, $10 \mu \mathrm{m}$. (K) Nrf2 mRNA levels in primary neurons transduced with aSyn AAV and lentiviral scRNA or shNrf2 RNA. (L) LDH release in primary neurons transduced with aSyn AAV and lentiviral scRNA or shNrf2 RNA and treated with PBS or NDP-MSH. (M) MAP2 staining and $(\mathrm{N})$ quantification of MAP2-postive cells in primary neurons transduced with aSyn AAV and lentiviral scRNA or shNrf2 RNA. Scale bar, $10 \mu \mathrm{m}$. Visual field area $=0.13508 \mathrm{~mm}^{2} .{ }^{*} P<0.05$, ${ }^{\star \star} P<0.01,{ }^{* \star \star} P<0.001, \mathrm{~ns}=$ not statistically significant. $\mathrm{n}=3$ replicates. Experiments were repeated $\geq 3$ times. 

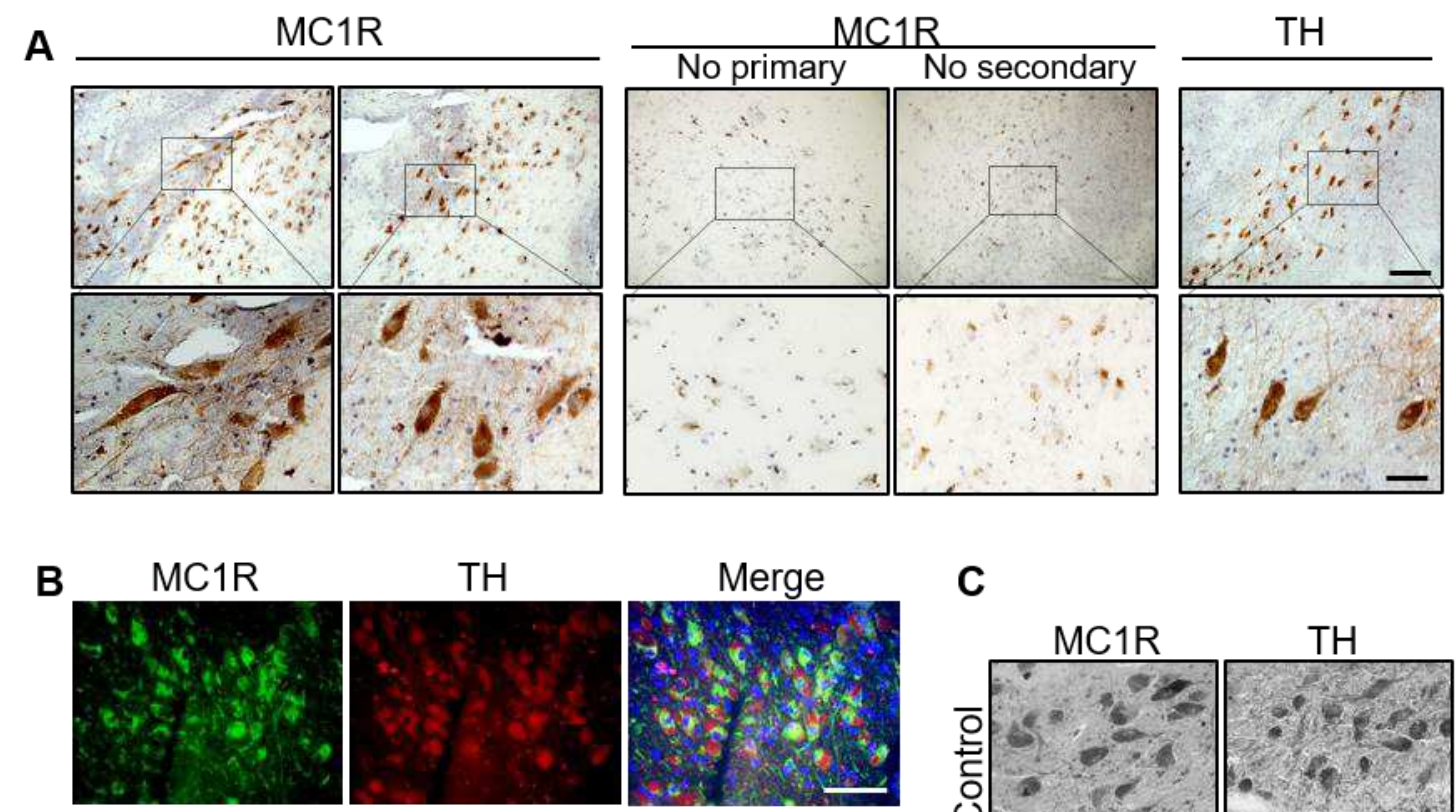

$\mathrm{TH}$

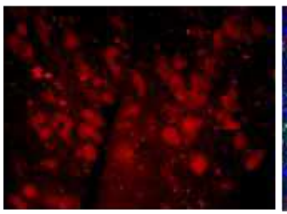

Merge

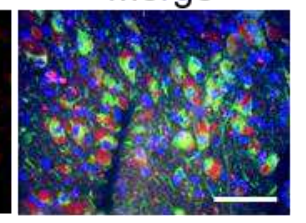

MC1R

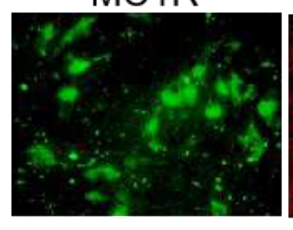

GFAP

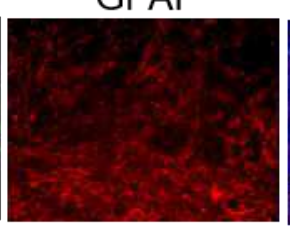

Merge

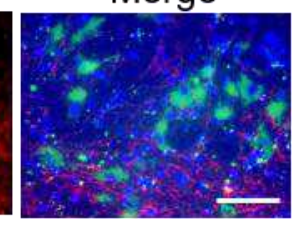

C
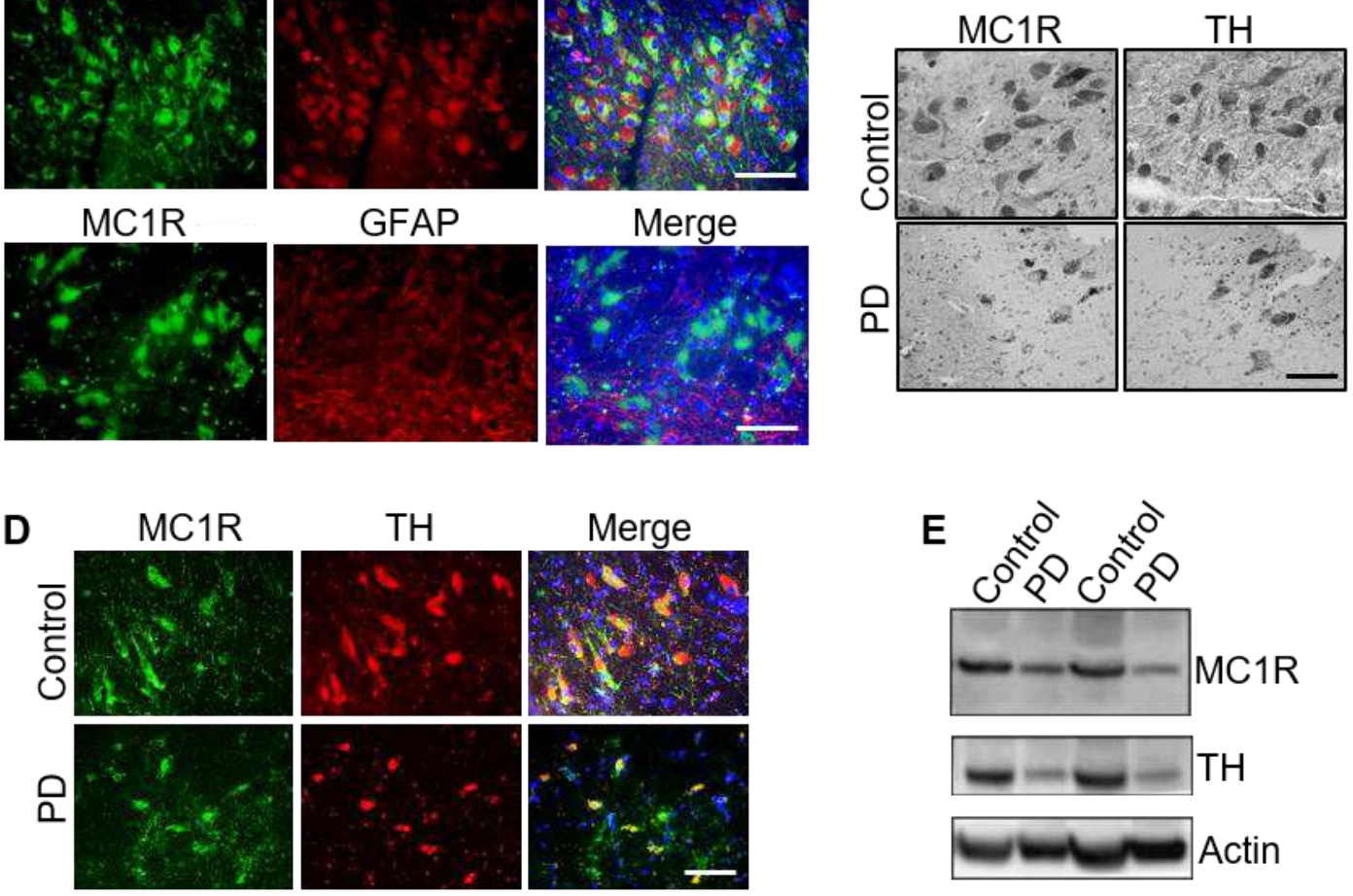

Fig. 6. MC1R is present in dopaminergic neurons in humans and is reduced in patients with

PD. (A) Immunohistochemistry for MC1R and TH in the SN of a 60-year-old control individual, PMI $15 \mathrm{~h}$. Control sections were processed in the same manner, except primary or secondary antibodies were omitted. Scale bars, top $100 \mu \mathrm{m}$, bottom $25 \mu \mathrm{m}$. (B) Fluorescence double-staining for MC1R and TH or GFAP in the SN of a 63-year-old control individual, PMI $16 \mathrm{~h}$. Scale bar, $50 \mu \mathrm{m}$. (C) Immunohistochemistry for MC1R and TH in the SN of a 91-year-old control individual, PMI $8 \mathrm{~h}$, and an 84-year-old PD patient, PMI 6 h. Scale bar, $50 \mu \mathrm{m}$. (D) Fluorescence double-staining of MC1R and $\mathrm{TH}$ in the SN of an 87-year-old control individual, PMI $48 \mathrm{~h}$, and a 91-year-old PD patient, PMI $32 \mathrm{~h}$. Scale bar, $50 \mu \mathrm{m}$. (E) Immunoblot for MC1R and TH using SN tissue from control individuals 
and PD patients. Actin as a loading control. Age (years)/PMI (h): 91/8 and 60/15 for control individuals, 84/6 and 69/17 for PD patients. 


\title{
Supplementary Information for
}

\section{Melanocortin 1 receptor activation protects against alpha-synuclein pathologies in models of Parkinson's Disease}

\author{
Waijiao Cai ${ }^{1}$, Danielle Feng ${ }^{1}$, Yue Lin ${ }^{1}$, Charles R. Vanderburg ${ }^{1,2}$, Yuehang Xu${ }^{1}$, Pamela Mclean ${ }^{3}$, \\ Matthew P. Frosch ${ }^{1,2,4}$, David E. Fisher ${ }^{5}$, Michael A. Schwarzschild ${ }^{1}$, Xiqun Chen $^{1 *}$ \\ ${ }^{1}$ MassGeneral Institute for Neurodegenerative Disease, Department of Neurology, Massachusetts \\ General Hospital, Harvard Medical School, Boston, USA. \\ ${ }^{2}$ Harvard NeuroDiscovery Advanced Tissue Resource Center, Massachusetts General Hospital, \\ Harvard Medical School, Boston, USA. \\ ${ }^{3}$ Mayo Clinic, Jacksonville, Florida, USA. \\ ${ }^{4}$ Neuropathology Service, Massachusetts General Hospital, Harvard Medical School, Boston, USA. \\ ${ }^{5}$ Cutaneous Biology Research Center, Department of Dermatology, Massachusetts General \\ Hospital, Harvard Medical School, Boston, USA.
}


A

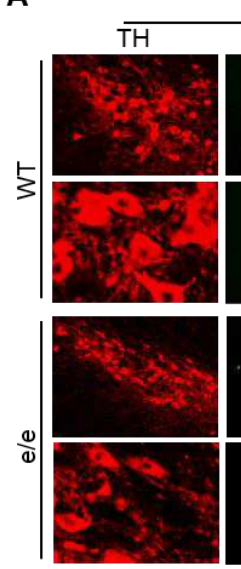

C
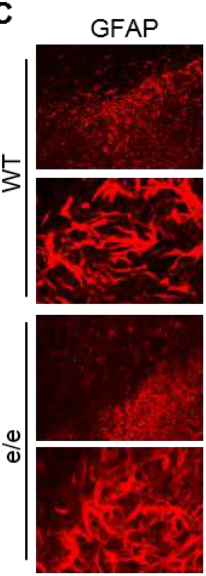

Contra
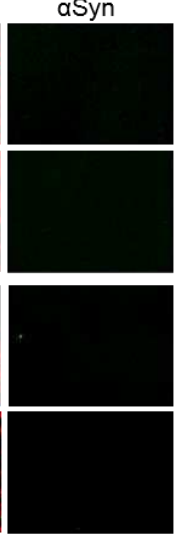

aSyn
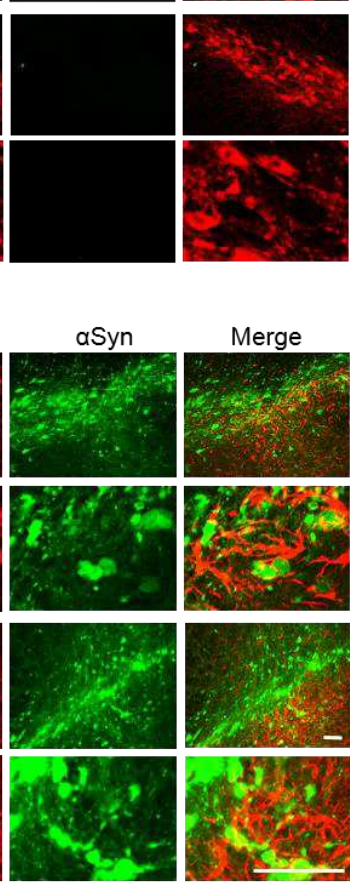
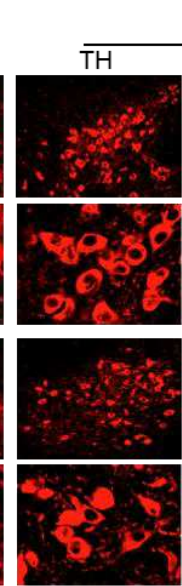

D
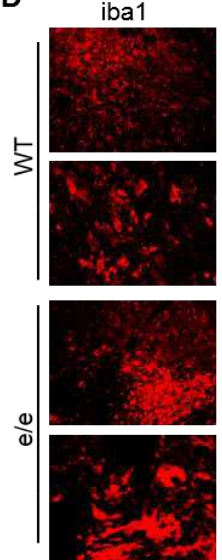

B
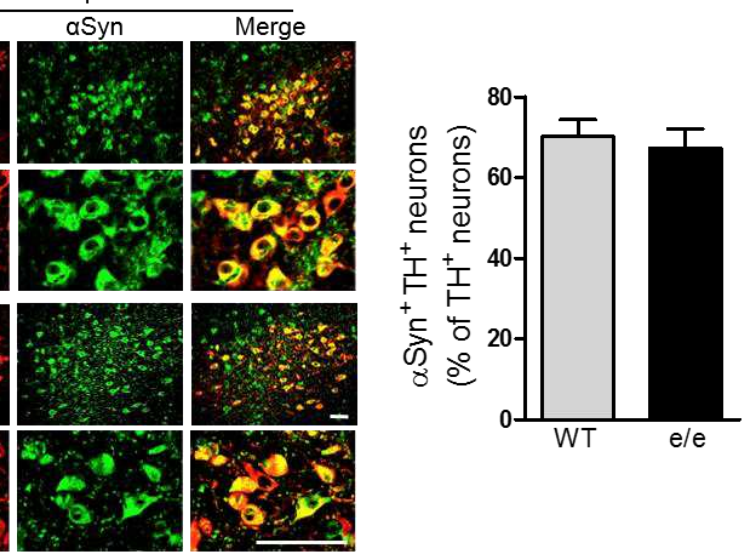

Fig. S1. Human aSyn transduction in dopaminergic neurons and glial and microglial cells in

the SN. MC1 $R^{\mathrm{e} / \mathrm{e}}$ and WT mice were injected unilaterally with human aSyn AAV into the SN and sacrificed 4 weeks later. (A) Immunofluorescence double-staining for TH and human aSyn. Scale bars, $50 \mu \mathrm{m}$. (B) Percentage of aSyn-transduced dopaminergic neurons in the ipsilateral SN. $n=5$ mice/group. (C) Immunofluorescence double-staining for GFAP and human aSyn on the ipsilateral side. Scale bars, $50 \mu \mathrm{m}$. (D) Immunofluorescence double-staining for iba1 and human aSyn on the ipsilateral side. Scale bars, $50 \mu \mathrm{m}$. 
A Venus
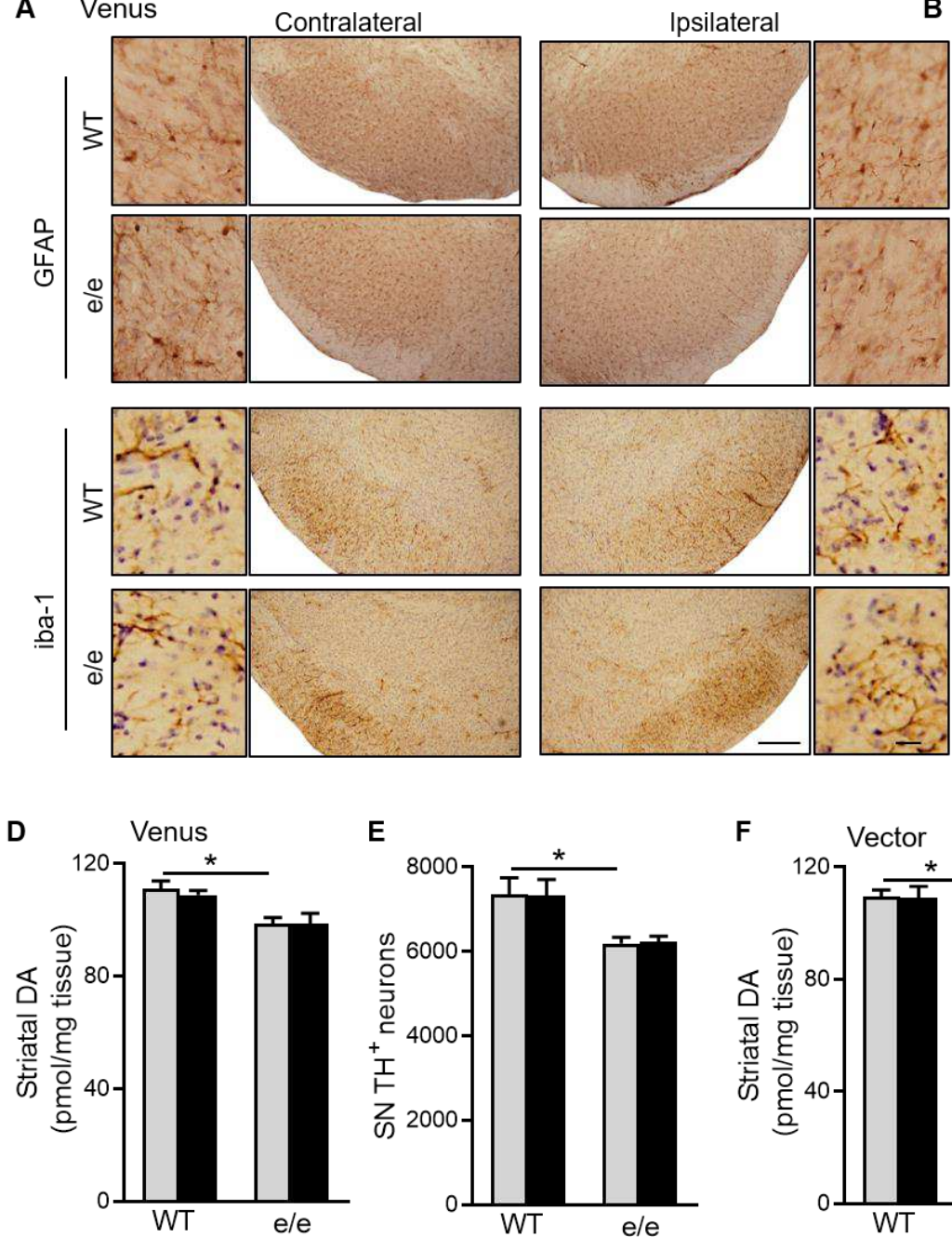

E

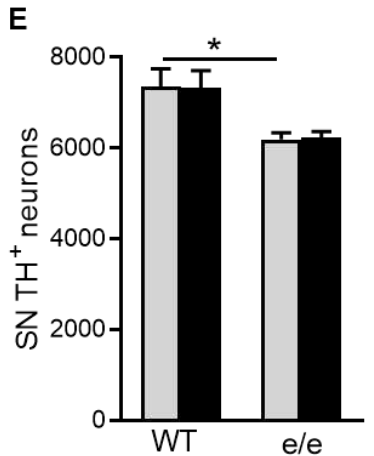

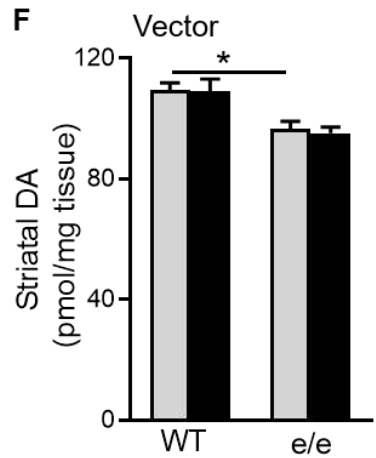

Fig. S2. Venus or vector did not induce astrogliosis, microgliosis or dopaminergic neuron

loss in the SN. $M C 1 R^{e / e}$ and WT mice were injected unilaterally with venus or vector into the SN and sacrificed 12 weeks later. (A) GFAP or iba-1 immunostaining in the SN. Scale bars, lower magnification $100 \mu \mathrm{m}$, higher magnification $10 \mu \mathrm{m}$. (B) quantification of integrated optical density of GFAP in the SN. n=4 mice/group. (C) Morphological classification and quantification of iba1positive cells in the SN. $n=4$ mice/group. (D) Striatal dopamine content and (C) stereological quantification of TH-positive in the $\mathrm{SN}$ with venus injection. $\mathrm{n}=6$ mice/group. (F) Striatal dopamine content and (G) stereological quantification of $\mathrm{TH}$-positive in the $\mathrm{SN}$ with empty vector injection. $\mathrm{n}=6$ mice/group. ${ }^{*} \mathrm{P}<0.05$. 


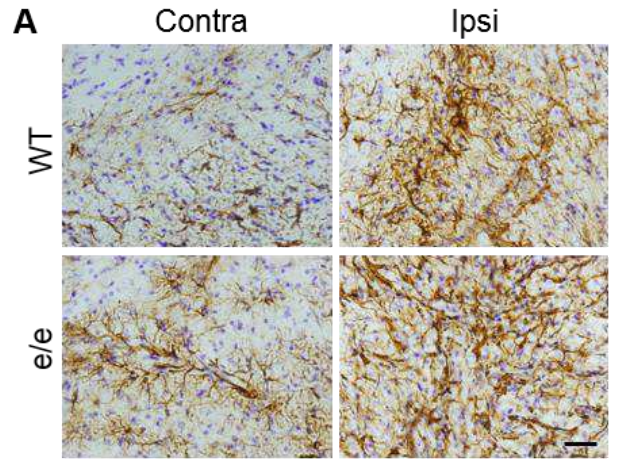

B

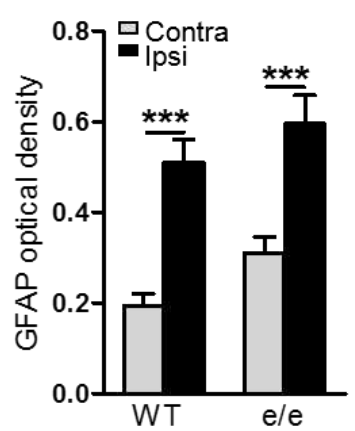

C

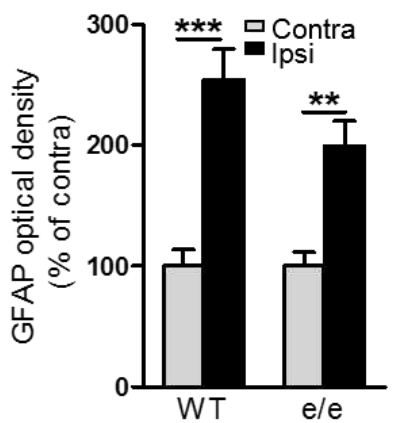

D
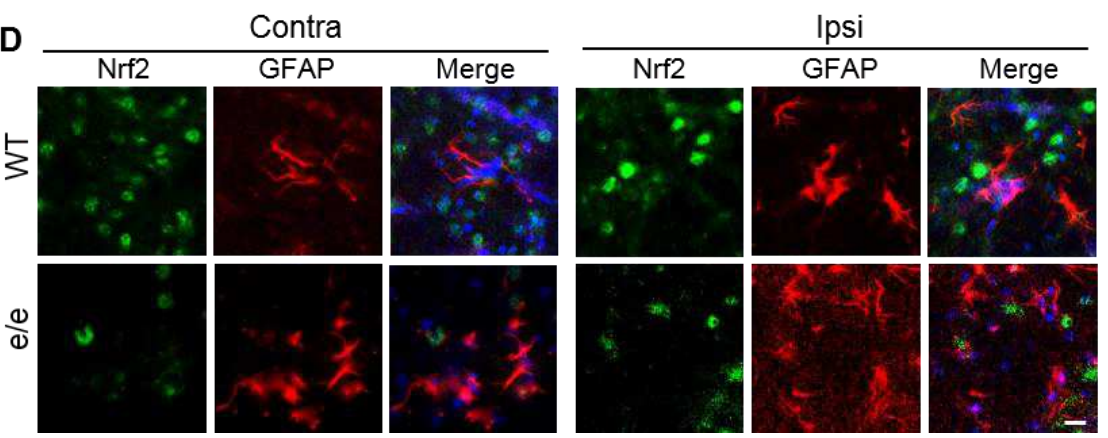

Fig. S3. aSyn-induced astrogliosis and Nrf2 expression in astrocytes. $M C 1 R^{e / e}$ and WT mice were injected unilaterally with human WT aSyn AAV into the SN and sacrificed 12 weeks later. (A) GFAP staining in the SN and (B) quantification of integrated optical density in original values or (C) normalized to contralateral values. $n=6$ mice/group. Scale bar, $25 \mu \mathrm{m}$. (D) Immunofluorescence double-staining of Nrf2 and GFAP 4 weeks post-AAV injection. Scale bar, $10 \mu \mathrm{m} .{ }^{* *} P<0.01$, ${ }^{* * *} P<0.001$. 

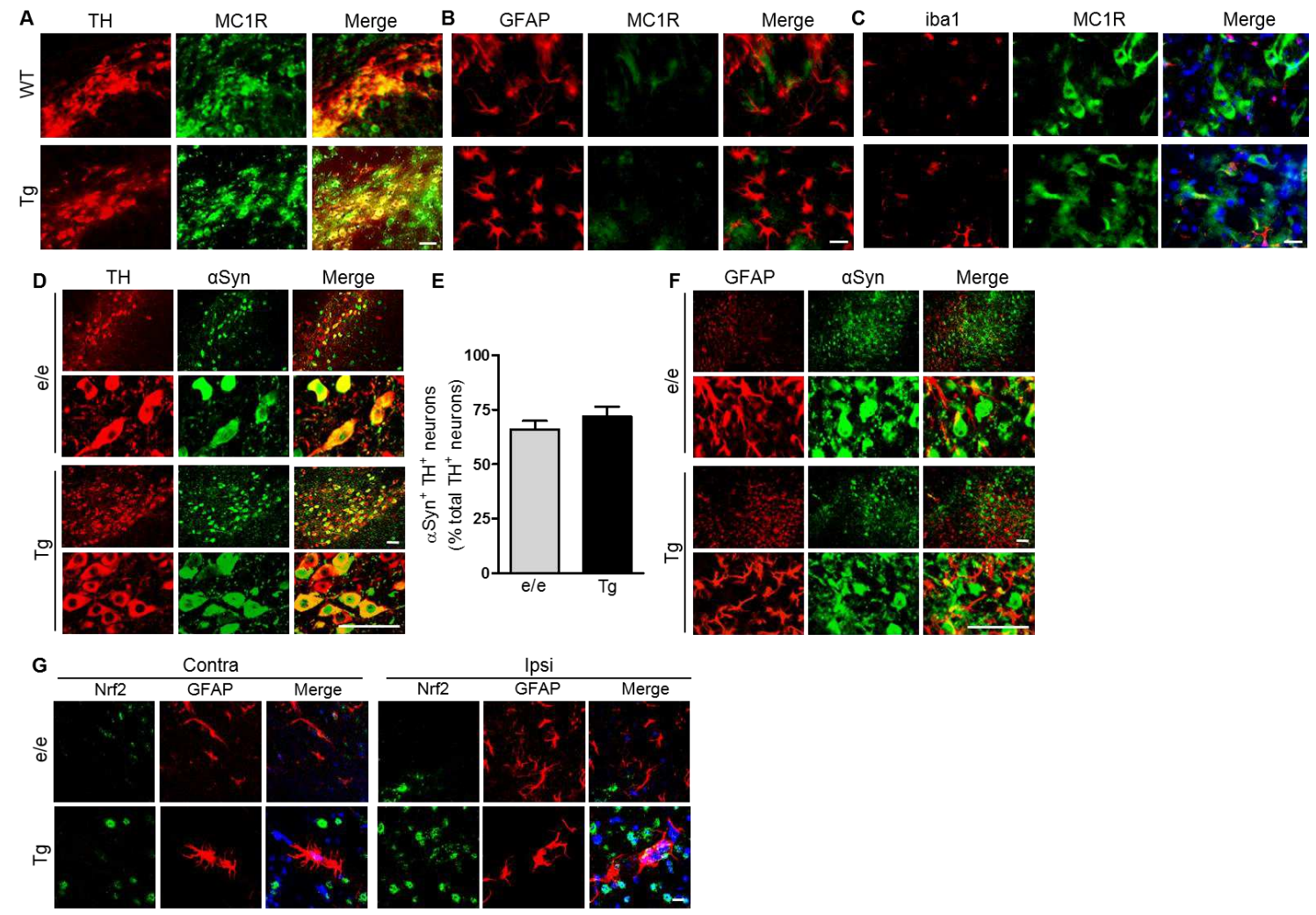

Fig. S4. Tg MC1R expression, aSyn transduction, and Nrf2 expression in the SN $M C 1 R^{e / e} \mathrm{Tg}$ mice. (A) Immunofluorescence double-staining for TH and MC1R. Scale bar, $50 \mu \mathrm{m}$. (B) Immunofluorescence double-staining for GFAP and MC1R. Scale bar, $10 \mu \mathrm{m}$. (C) Immunofluorescence double-staining for iba1 and MC1R. Scale bar, $10 \mu \mathrm{m}$. $M C 1 R^{\mathrm{e} / \mathrm{T}} \mathrm{Tg}$ and $M C 1 R^{e / e}$ mice were injected unilaterally with human aSyn AAV into the SN. (D) Immunofluorescence double-staining for $\mathrm{TH}$ and human aSyn and (E) percentage of aSyntransduced dopaminergic neurons 4 weeks post-AAV injection. $n=4$ mice/ group. Scale bars, 50 $\mu \mathrm{m}$. (F) Immunofluorescence double-staining of GFAP and human aSyn 4 weeks post-AAV injection. Scale bars, $50 \mu \mathrm{m}$. (G) Immunofluorescence double-staining of Nrf2 and GFAP 4 weeks post-AAV injection. Scale bar, $10 \mu \mathrm{m}$. 

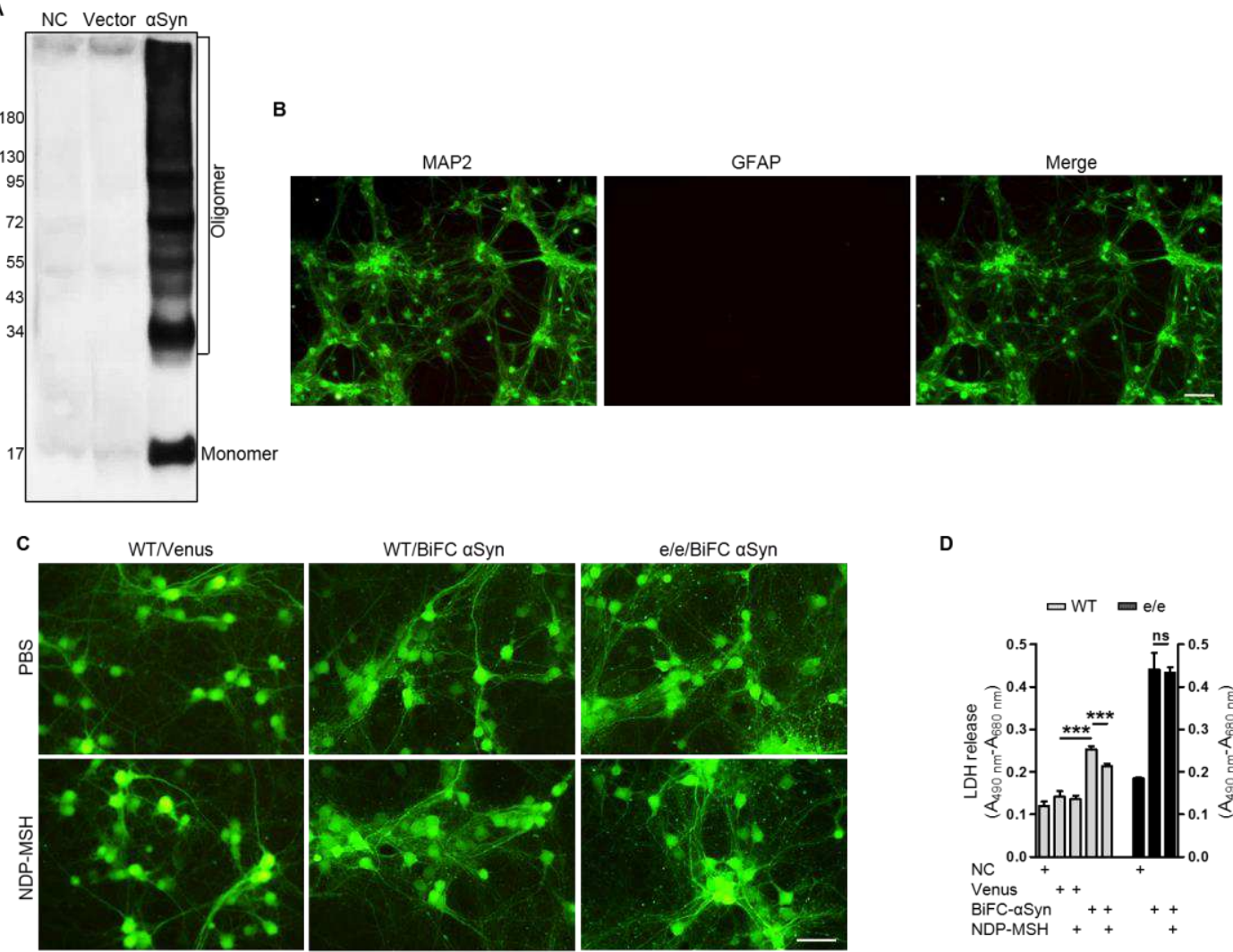

D

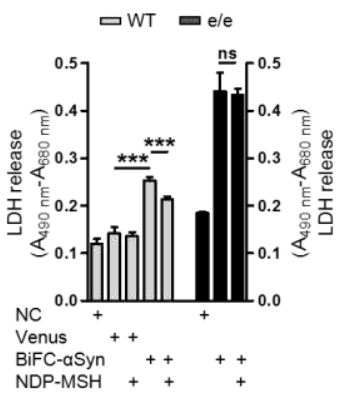

Fig. S5. aSyn species in HEK293T cells overexpressing human WT aSyn; characterization of primary cortical neurons and protection of MC1R activation against $\alpha$ Syn cytotoxicity. (A) Immunoblot for oligomeric and monomeric aSyn in HEK293T cells transfected with human WT aSyn or control vector. NC: non-transfected control cells. (B) MAP2 and GFAP staining in WT primary cortical neurons at DIV5. Scale bar, $25 \mu \mathrm{m}$. (C) aSyn oligomerization visualized by fluorescence at DIV9 in WT or MC1 $R^{e / e}$ cortical neurons transduced with BiFC aSyn AAV or control venus AAV. Scale bar, $25 \mu \mathrm{m}$. (D) LDH release at DIV12 in WT or $M C 1 R^{\mathrm{e} / \mathrm{e}}$ cortical neurons transduced with BiFC aSyn or venus AAV and treated with NDP-MSH. ${ }^{* \star} P<0.001$. ns $=$ not statistically significant. 
Supplementary Table 1: Primers used for qPCR

\begin{tabular}{|c|c|}
\hline Gene name & Primer sequence \\
\hline \multicolumn{2}{|l|}{ Mouse } \\
\hline \multirow[t]{2}{*}{ IL-6 } & F: TAGTCCTTCCTACCCCAАTTTCC \\
\hline & R: TTGGTCCTTAGCCACTCCTTC \\
\hline \multirow[t]{2}{*}{$T N F-\alpha$} & F: СССТСАСАСТСАGАТСАТСТTCT \\
\hline & R: GCTACGACGTGGGCTACAG \\
\hline \multirow[t]{2}{*}{ ICAM1 } & F: TCAGTGGCTGAAAGATGAGC \\
\hline & R: CGGAAACGAATACACGGTGA \\
\hline \multirow[t]{2}{*}{$I L-1 \alpha$} & F: GACAGGGAACTTAGGGAGCA \\
\hline & R: TTGGCCATCTTGATTTCAGAGT \\
\hline \multirow[t]{2}{*}{$\mathrm{HO}-1$} & F: GCTACCTGGGTGACCTCTCA \\
\hline & R: GCAGCTCCTCAAACAGCTCAAT \\
\hline \multirow[t]{2}{*}{$N Q O-1$} & F: CCTTTCCAGAATAAGAAGACC \\
\hline & R: AATGCTGTAAACCAGTTGAG \\
\hline \multirow[t]{2}{*}{ GCLC } & F: CTATCTGCCCAATTGTTATGG \\
\hline & R: ACAGGTAGCTATCTATTGAGTC \\
\hline \multirow[t]{2}{*}{$G C L M$} & F: GCACAGGTAAAACCCAATAG \\
\hline & R: TTAGCAAAGGCAGTCAAATC \\
\hline \multirow[t]{2}{*}{ GAPDH } & F: CATGGCCTTCCGTGTTCCTA \\
\hline & R: CCTGCTTCACCACCTTCTTGAT \\
\hline \multicolumn{2}{|l|}{ Human } \\
\hline \multirow[t]{2}{*}{ Nrf2 } & F: AGTGGATCTGCCAACTACTC \\
\hline & R: CATCTACAAACGGGAATGTCTG \\
\hline \multirow[t]{2}{*}{$\mathrm{HO}-1$} & F: ACTGCGTTCCTGCTCAACATC \\
\hline & R: GCTCTGGTCCTTGGTGTCATG \\
\hline \multirow[t]{2}{*}{ GAPDH } & F: CATGAGAAGTATGACAACAGCCT \\
\hline & R: AGTCCTTCCACGATACCAAAGT \\
\hline
\end{tabular}




\section{Figures}
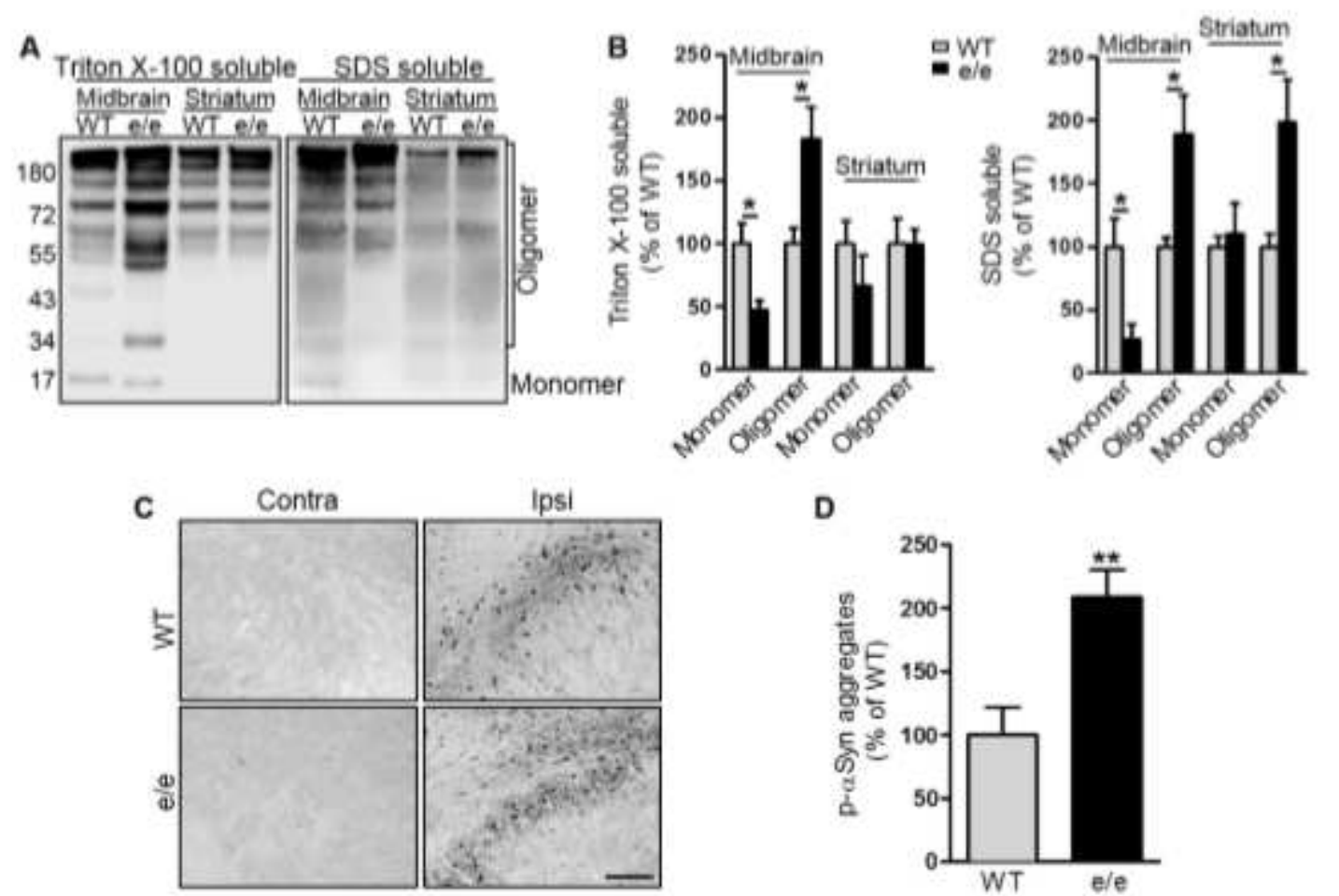

D
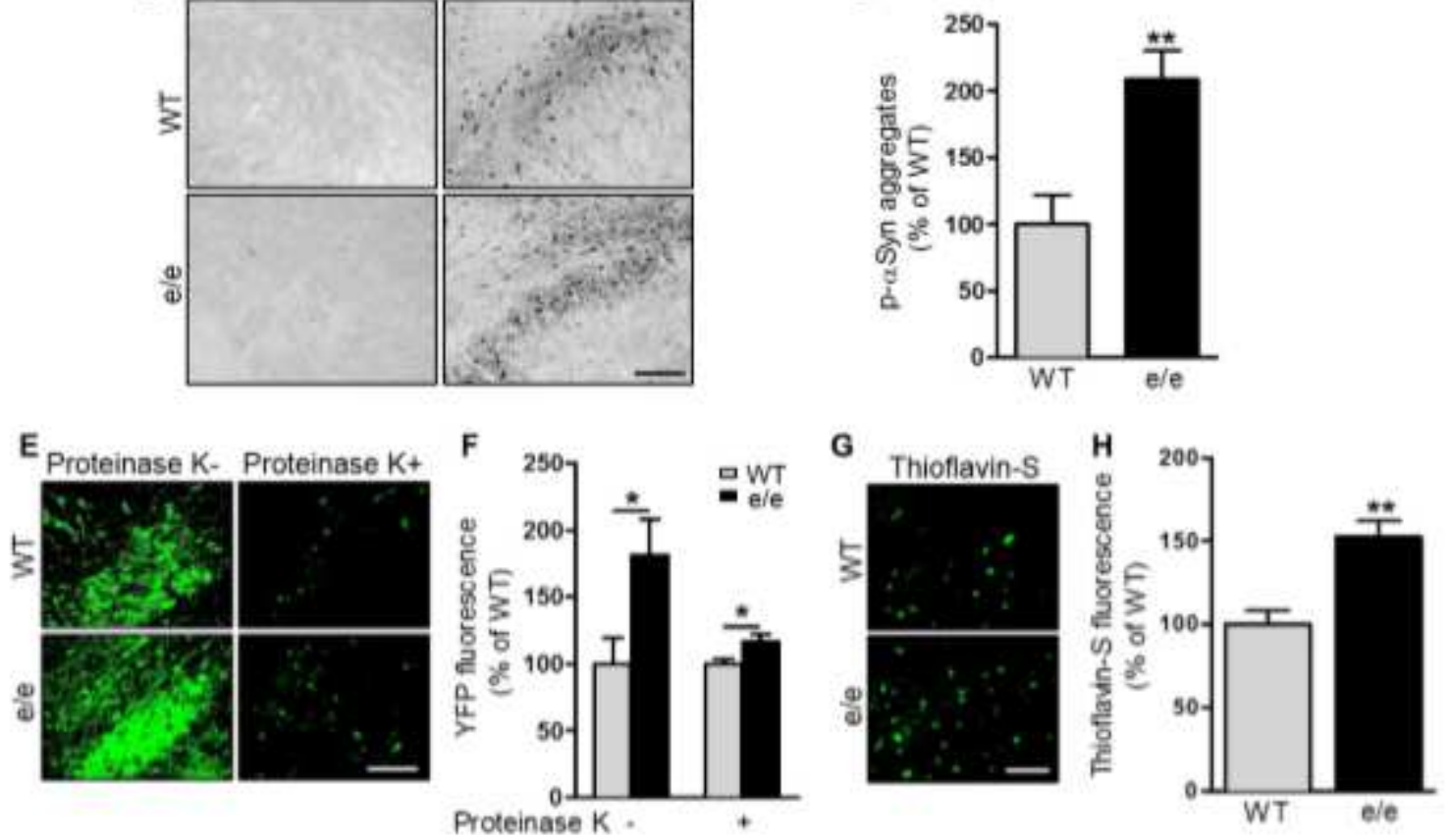

Figure 1

MC1R disruption exacerbates synucleinopathies in the nigrostriatal pathway in an aSyn AAV mouse model. MC1Re/e and WT mice were injected unilaterally with human WT aSyn AAV into the SN and sacrificed 8 weeks later: (A) Immunoblot of human aSyn species in Triton X 100-soluble and -insoluble SDS-soluble fractions in ipsilateral ventral midbrain and striatum and (B) quantification of aSyn monomers and oligomers. $n=3$ mice/group. (C) p-aSyn staining and (D) quantification of $p$-aSyn aggregates in the ipsilateral SN. $\mathrm{n}=3$ mice/group. Scale bar, $50 \mu \mathrm{m}$. MC1Re/e and WT mice were injected unilaterally with BiFC human WT aSyn AAV into the SN and sacrificed 8 weeks later: (E) VenusYFP fluorescence before and after proteinase $\mathrm{K}$ treatment and $(\mathrm{F})$ quantification of venusYFP fluorescence density in the ipsilateral SN. n=4 mice/group. Scale bar, $50 \mu \mathrm{m}$. (G) Thioflavin-S staining and $(\mathrm{H})$ quantification of thioflavin-S fluorescence density in the ipsilateral SN. $\mathrm{n}=4$ mice/group. Scale bar, $50 \mu \mathrm{m}$. *P<0.05, **P $<0.01$. 

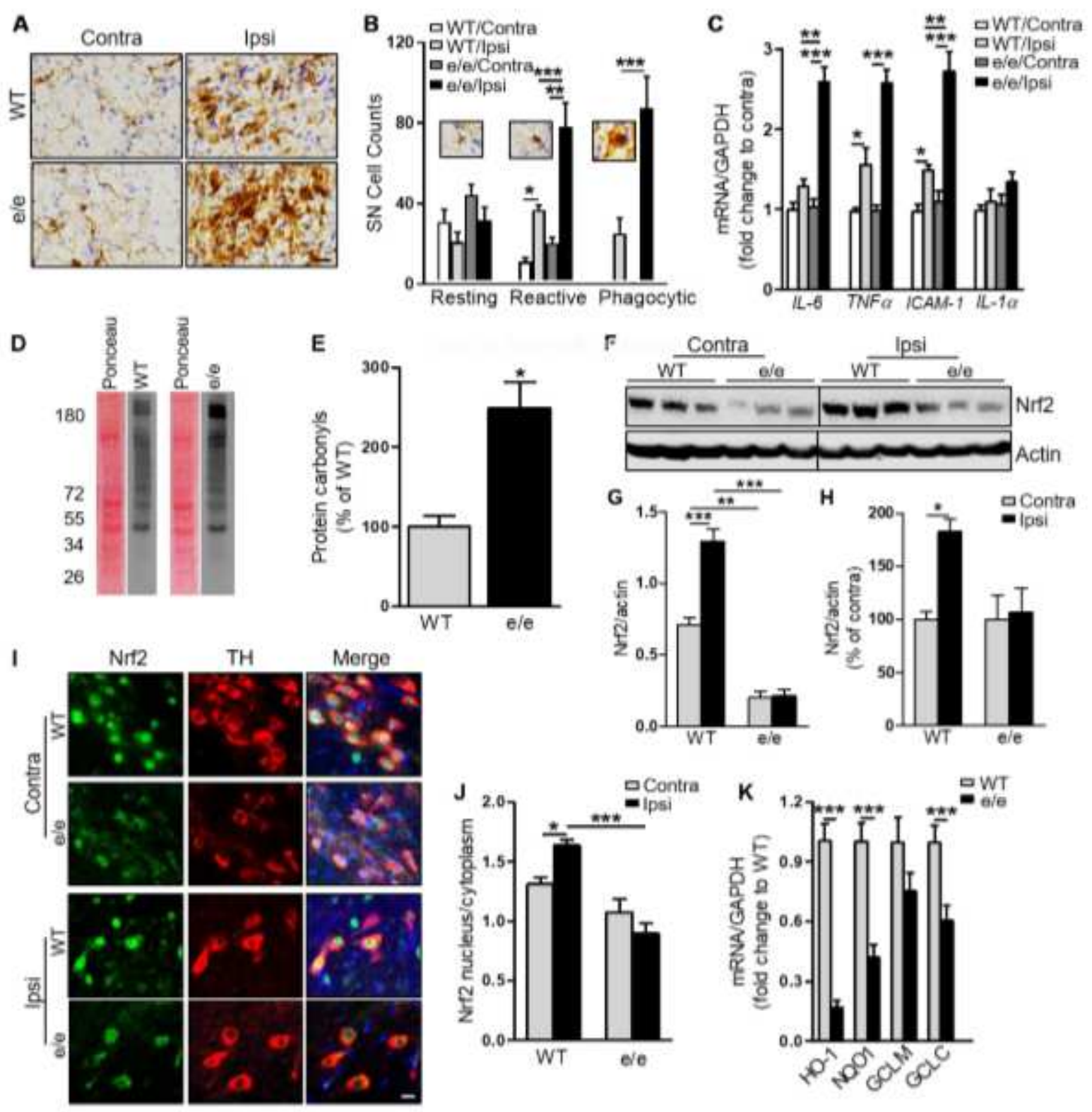

\section{Figure 2}

MC1R disruption amplifies microglia activation and alters Nrf2 response to aSyn overexpression in the nigrostriatal pathway. MC1Re/e and WT mice were injected unilaterally with human WT aSyn AAV into the SN and sacrificed 8 weeks later: (A) Iba1 staining and (B) morphological classification and quantification of iba1-positive cells in the SN. $n=4$ mice/group. Scale bar, $30 \mu \mathrm{m}$. (C) IL-1a, IL-6, TNFa, and ICAM1 mRNA levels in ventral midbrain. $n=5$ mice/group. (D) Representative oxyblots for protein carbonyls and the corresponding Ponceau $S$ staining in the ipsilateral ventral midbrain and $(E)$ quantification of band density. $n=3$ mice/group. (F) Immunoblot for Nrf2 using ventral midbrain tissue and $(\mathrm{G})$ quantification of Nrf2 band density in original values or $(\mathrm{H})$ normalized to contralateral side values. $n=3$ mice/group. (I) SN sections double-stained for Nrf2 and TH and (J) quantification of nuclear and cytoplasmic Nrf2. n=3 mice/group. Scale bar, $20 \mu \mathrm{m}$. (K) mRNA levels of Nrf2 target genes HO-1, NQ01, GCLM, and GCLC in the ipsilateral ventral midbrain. $n=5$ mice/group. ${ }^{*} P<0.05,{ }^{* *} P<0.01$, $\star * \star P<0.001$. 

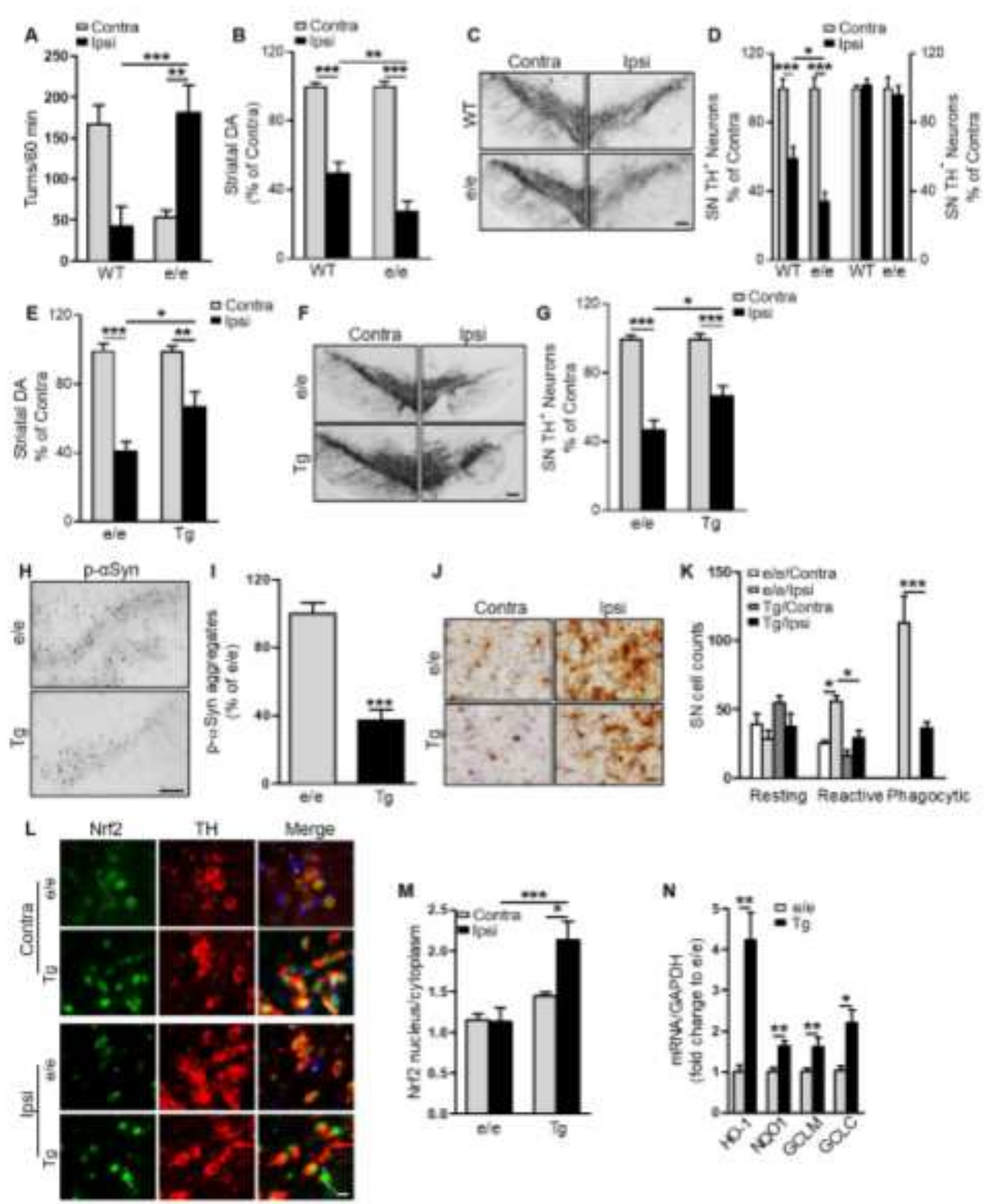

\section{Figure 3}

aSyn-induced dopaminergic neurotoxicity is exacerbated by MC1R disruption and reversed by human MC1R transgene. MC1Re/e and WT mice were injected unilaterally with human WT aSyn AAV into the SN. (A) Contralateral and ipsilateral turns induced by amphetamine 12 weeks post-AAV injection. $n=12$ mice/group. (B) Striatal dopamine content 16 weeks post-AAV injection. $n=12$ mice/group. (C) TH staining and (D) stereological quantification of TH-positive and -negative cells in the SN 16 weeks postAAV injection. $\mathrm{n}=12$ mice/group. Scale bar, $100 \mu \mathrm{m}$. MC1Re/eTg and MC1Re/e mice were injected unilaterally with human WT aSyn AAV into the SN. (E) Striatal dopamine content 16 weeks post-AAV injection. $n=6-7$ mice/group. (F) TH staining and (G) stereological quantification of TH-positive and negative cells in the SN 16 weeks post-AAV injection. $n=6-7$ mice/group. Scale bar, $100 \mu m$. (H) p-aSyn staining and (I) quantification of p-aSyn aggregates in the ipsilateral SNpc 12 weeks post-AAV injection. $\mathrm{n}=4$ mice/group. Scale bar, $50 \mu \mathrm{m}$. (J) Iba1 staining and morphological classification and (K) quantification of iba1-positive cells in the SNpc 12 weeks post-AAV injection. $n=4$ mice/group. Scale bar, 
$30 \mu \mathrm{m}$. (L) Nrf2 and TH double labeling and (M) quantification of nuclear and cytoplasmic Nrf2 in the SNpc 12 weeks post-AAV injection. $n=4$ mice/group. Scale bar, $20 \mu \mathrm{m}$. (N) mRNA levels of Nrf2 target genes HO-1, NQ01, GCLC, and GCLM in the ipsilateral ventral midbrain 12 weeks post-AAV injection. $\mathrm{n}=5$ mice/group. ${ }^{*} P<0.05,{ }^{\star *} P<0.01,{ }^{\star} * \star P<0.001$. Values in $B, D, E, G$ are presented as percentages of their own contralateral mean values.
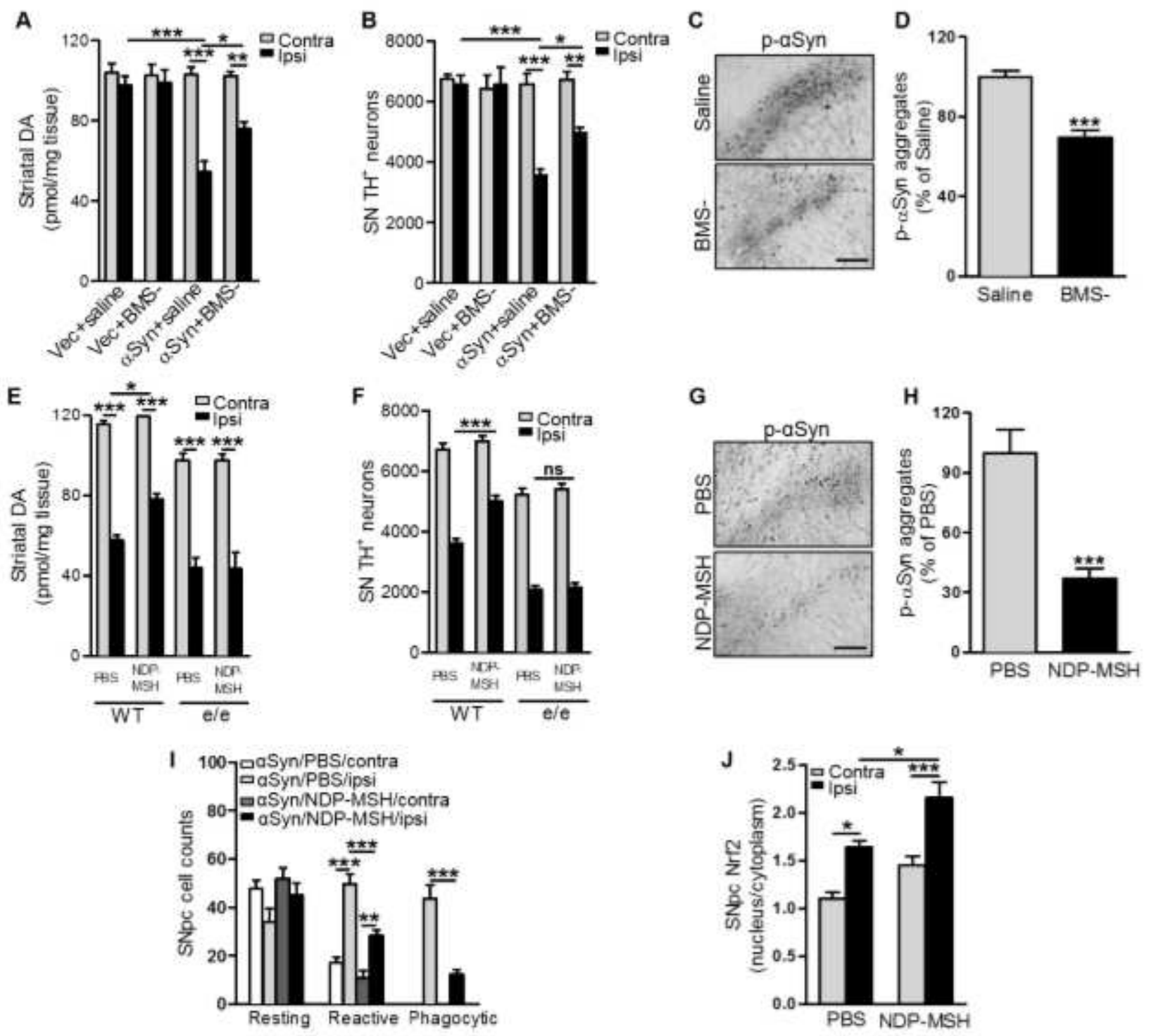

\section{Figure 4}

MC1R agonists protect against aSyn-induced dopaminergic neurotoxicity. C57BI/6J mice were injected with aSyn or empty vector (Vec) AAV, treated subcutaneously with $20 \mathrm{mg} / \mathrm{kg}$ BMS-470539 (BMS-) or saline for 4 weeks, and sacrificed 16 weeks post-AAV injection. (A) Striatal dopamine content. $n=6-8$ mice/group. (B) Stereological quantification of TH-positive cells in the SN. n=6-8 mice/group. (C) p-aSyn staining and (D) quantification of p-aSyn aggregates in the ipsilateral SN. $n=4$ mice/group. Scale bar, 50 $\mu \mathrm{m}$. WT and MC1Re/e mice were injected unilaterally with $3 \mathrm{nmol}$ NDP-MSH into the striatum and then with aSyn AAV into the SN. Mice were sacrificed 16 weeks post-AAV injection. (E) Striatal dopamine content. $n=7$ mice/group. (F) Stereological quantification of TH-positive cells in the SN. $n=7$ mice/group. 
(G) p-aSyn staining and $(\mathrm{H})$ quantification of p-aSyn aggregates in the ipsilateral SN. $n=4$ mice/group. Scale bar, $50 \mu \mathrm{m}$. (I) Morphological classification and quantification of iba1-positive cells in the SN in WT mice. $n=4$ mice/group. (J) Nuclear and cytoplasmic Nrf2 ratio in the SN in WT mice. $n=4$ mice/group. ${ }^{\star} \mathrm{P}<0.05,{ }^{* *} \mathrm{P}<0.01,{ }^{* \star *} \mathrm{P}<0.001$.
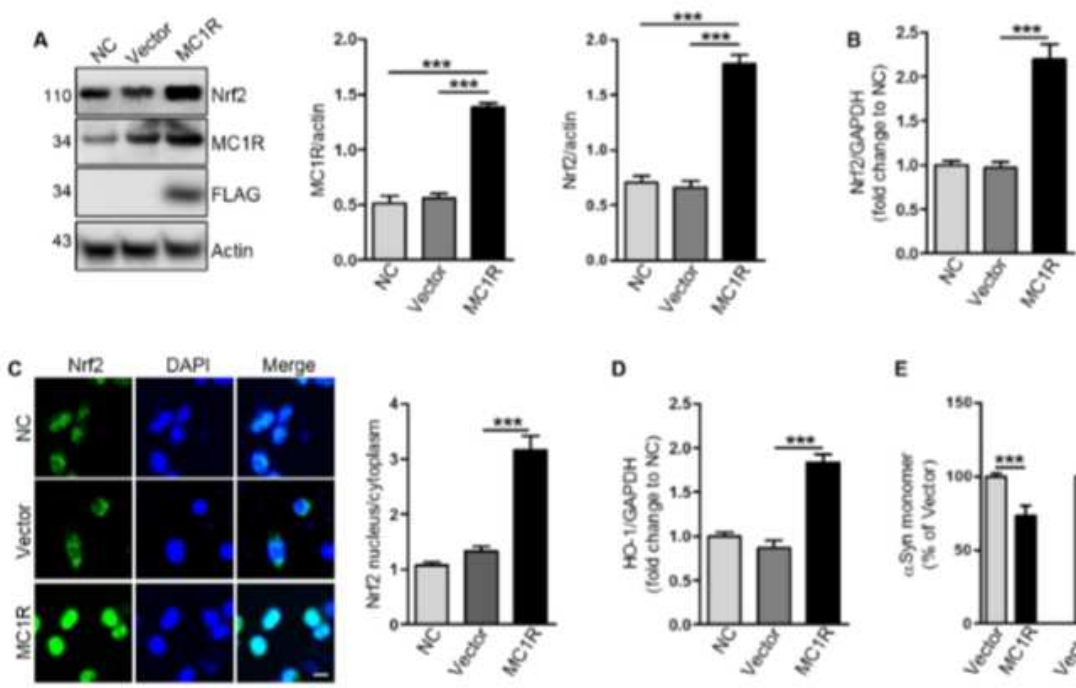

D

E
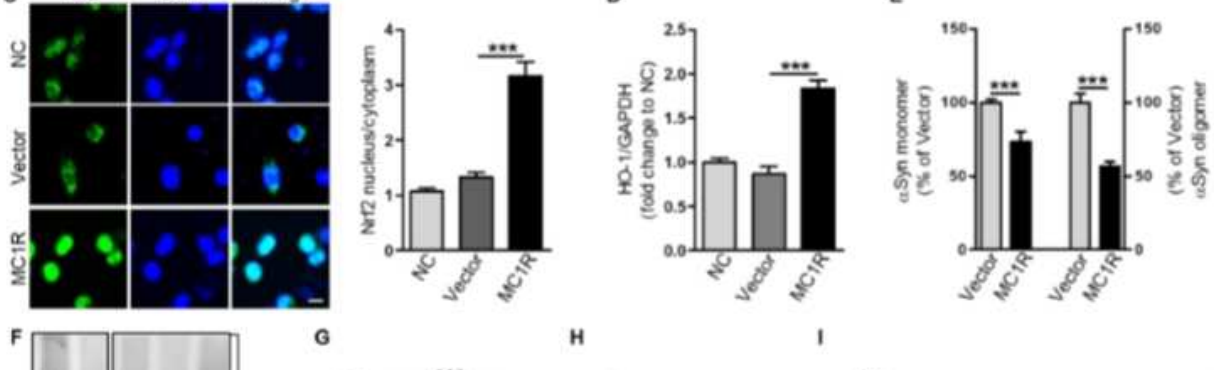

H
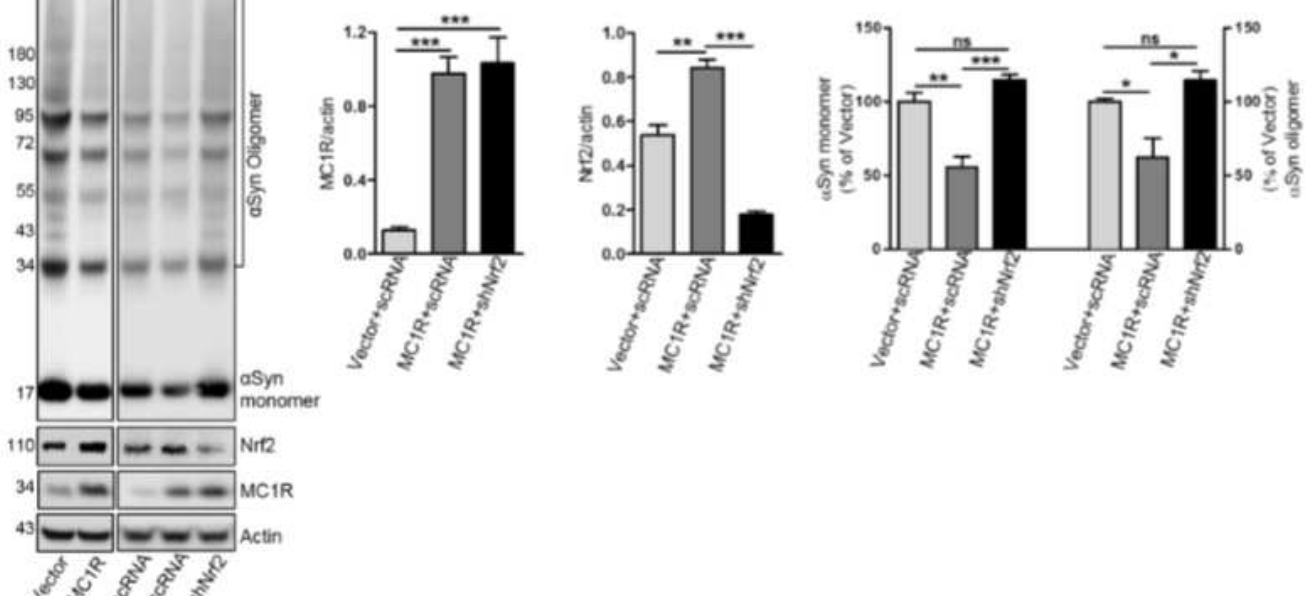

की

की

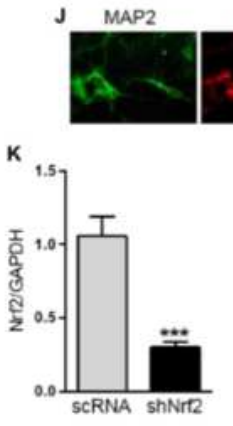

MC1R

Merge
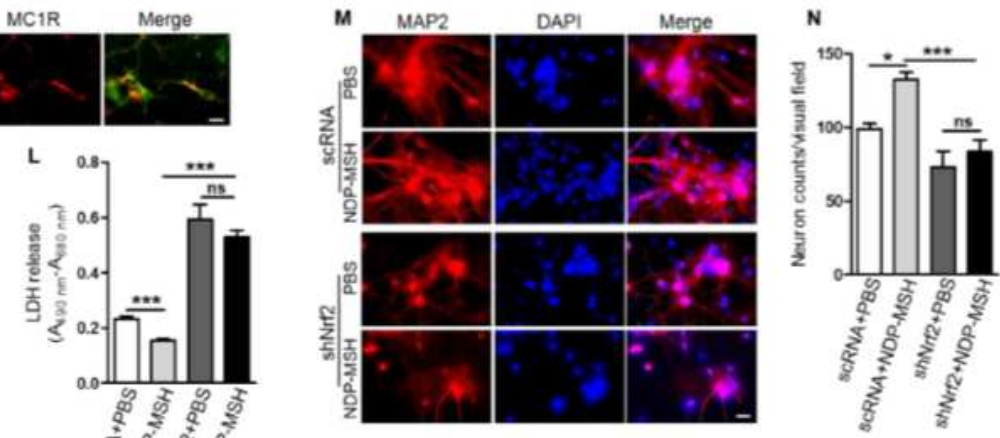

क्षे

Figure 5 
MC1R activation alleviates aSyn oligomerization and neurotoxicity by activating Nrf2 in vitro. HEK293T cells were co-transfected with aSyn and MC1R or vector (GPRC5A-Tango). Non-transfected cells served as controls (NC): (A) Immunoblot and quantification of MC1R and Nrf2. (B) Nrf2 mRNA levels. (C) Nrf2 staining and quantification of nuclear and cytoplasmic Nrf2. Nuclei were stained with DAPI. Scale bar, 10 $\mu \mathrm{m}$. (D) Nrf2 target gene HO-1 mRNA levels. (E and F) Immunoblot and quantification of aSyn species. HEK293T cells were transfected with aSyn, MC1R or vector control, and shNrf2 RNA or scRNA control: (F) Immunoblot and ( $\mathrm{G}$ and $\mathrm{H}$ ) quantification of MC1R and Nrf2. (F) Immunoblot and (I) quantification of aSyn species. Primary cortical neurons were prepared from embryonic day 16-17 WT mice: (J) MAP2 and MC1R staining at DIV5. Scale bar, $10 \mu \mathrm{m}$. (K) Nrf2 mRNA levels in primary neurons transduced with aSyn AAV and lentiviral scRNA or shNrf2 RNA. (L) LDH release in primary neurons transduced with aSyn AAV and lentiviral scRNA or shNrf2 RNA and treated with PBS or NDP-MSH. (M) MAP2 staining and (N) quantification of MAP2-postive cells in primary neurons transduced with aSyn AAV and lentiviral ScRNA or shNrf2 RNA. Scale bar, $10 \mu \mathrm{m}$. Visual field area $=0.13508 \mathrm{~mm} 2 .{ }^{*} \mathrm{P}<0.05,{ }^{\star *} \mathrm{P}<0.01,{ }^{\star \star \star *} \mathrm{P}<0.001$, ns=not statistically significant. $n=3$ replicates. Experiments were repeated $\geq 3$ times.

A
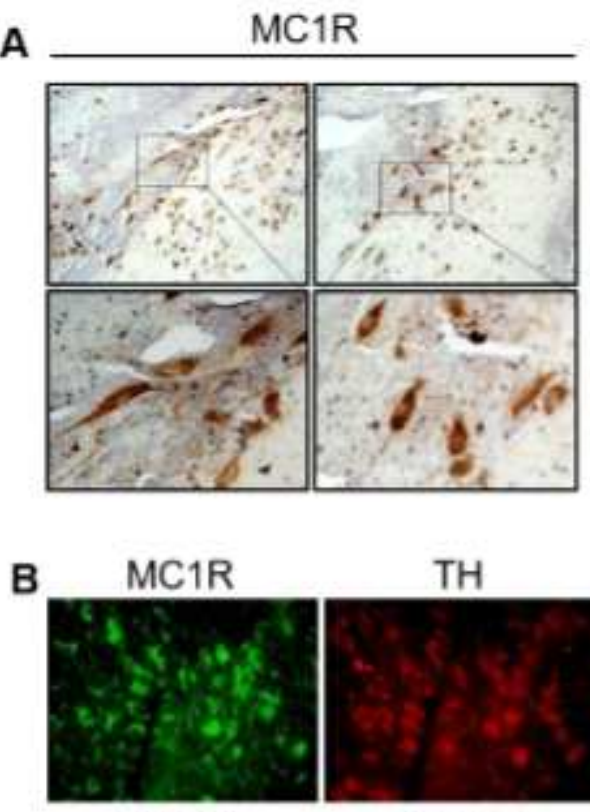

MC1R

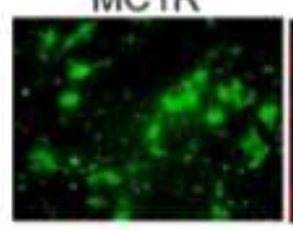

D
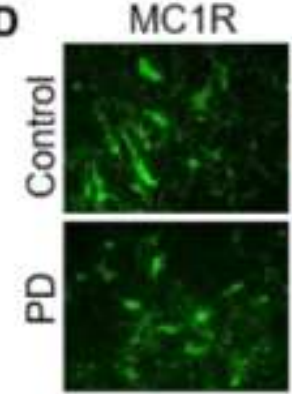

TH

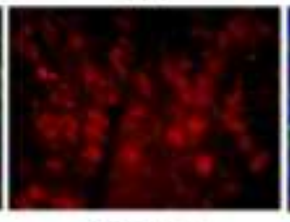

GFAP

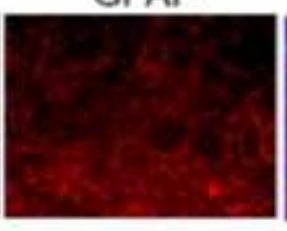

TH
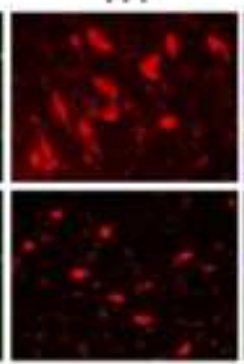
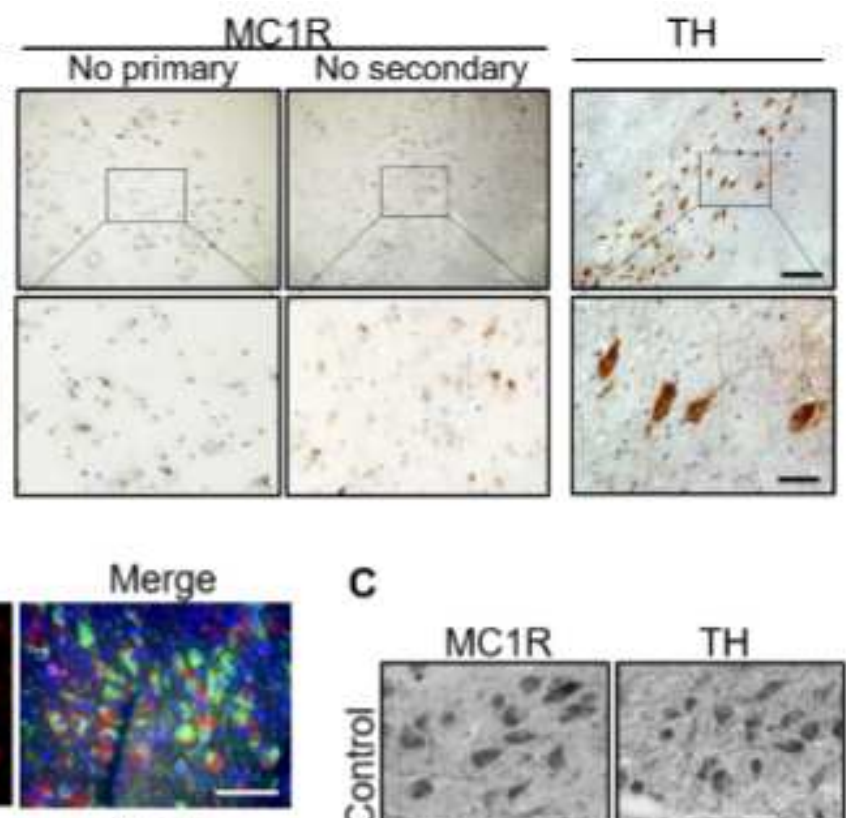

Merge

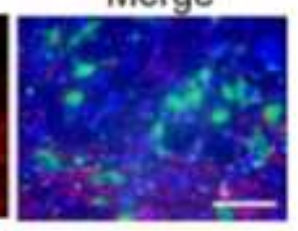

Merge
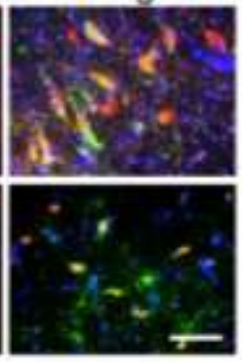

C

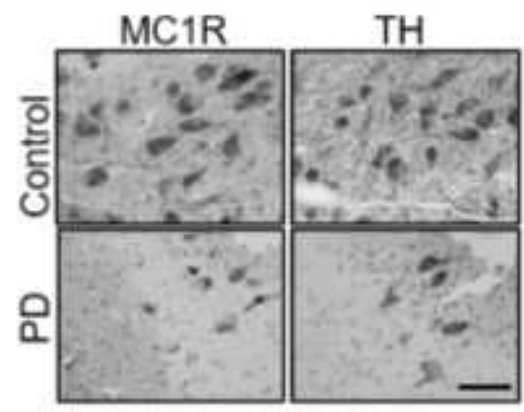

E

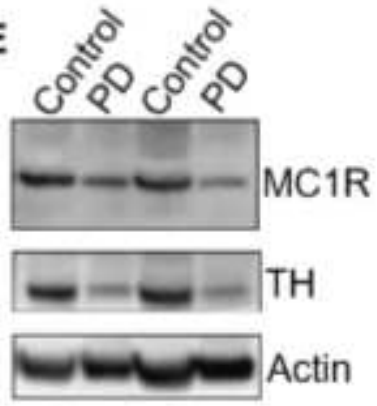




\section{Figure 6}

MC1R is present in dopaminergic neurons in humans and is reduced in patients with PD. (A) Immunohistochemistry for MC1R and TH in the SN of a 60-year-old control individual, PMI $15 \mathrm{~h}$. Control sections were processed in the same manner, except primary or secondary antibodies were omitted. Scale bars, top $100 \mu \mathrm{m}$, bottom $25 \mu \mathrm{m}$. (B) Fluorescence double-staining for MC1R and TH or GFAP in the SN of a 63-year-old control individual, PMI $16 \mathrm{~h}$. Scale bar, $50 \mu \mathrm{m}$. (C) Immunohistochemistry for MC1R and TH in the SN of a 91-year-old control individual, PMI 8 h, and an 84-year-old PD patient, PMI 6 h. Scale bar, 50 $\mu \mathrm{m}$. (D) Fluorescence double-staining of MC1R and TH in the SN of an 87-year-old control individual, PMI $48 \mathrm{~h}$, and a 91-year-old PD patient, PMI $32 \mathrm{~h}$. Scale bar, $50 \mu \mathrm{m}$. (E) Immunoblot for MC1R and TH using SN tissue from control individuals and PD patients. Actin as a loading control. Age (years)/PMI (h): 91/8 and $60 / 15$ for control individuals, $84 / 6$ and $69 / 17$ for PD patients.

\section{Supplementary Files}

This is a list of supplementary files associated with this preprint. Click to download.

- Supplmentery.pdf 